\title{
Piecewise uniform potential vorticity pancake shielded vortices
}

\author{
JEAN N. REINAUD \\ Mathematical Institute University of St Andrews, \\ North Haugh, St. Andrews, KY169SS, Scotland, UK \\ (Received 00 Month 20xx; final version received 00 Month 20xx)
}

\begin{abstract}
Shielded vortices consist of a core of potential vorticity of a given sign surrounded (or shielded) by a layer of opposite-signed potential vorticity. Such vortices have specific properties and have been the focus of numerous studies, first in two dimensional geometries (where potential vorticity is just the vertical component of the vorticity vector) and in geophysical applications (mostly in layered models). The present paper focuses on three-dimensional, spheroidal shielded vortices. In particular, we focus on vortical structures whose overall volume-integrated potential vorticity is zero. We restrict attention to vortices of piecewise uniform potential vorticity in the present research. We first revisit the problem within the quasi-geostrophic model, then we extend the results to the non-hydrostatic regime. We show that the stability of the structure depends on the ratio of potential vorticity between the inner core and the outer shield. In particular it depends on the polarity of the core and of the wavenumber of the azimuthal mode perturbed.
\end{abstract}

Keywords: Vortex dynamics; Vortex equilibria; Vortex stability

\section{Introduction}

Vortices not only abound in the oceans at all depths, but they are, together with jets, responsible for the transport of heat and tracers in the oceans at mesoscale. Recent estimates suggest that this part contributes up to half of the overall transport (see Zhang, Wang and Qiu 2014). The ubiquity of vortices in the oceans has been illustrated by many studies (see Ebbesmeyer et al. 1986, Chelton, Schlax and Samelson 2011, Chelton et al. 2007 and numerous other studies). Reviews on oceanic vortices may be found in Robinson (1983) and Carton (2001). In geophysical contexts, vortices may be conveniently defined as volumes of potential vorticity (hereinafter referred to as PV), a quantity materially conserved in absence of diabatic and viscous effects.

Three dimensional, quasi-geostrophic, vortices containing uniform PV have been the focus of several studies, in particular when the vortices are (initially) ellipsoidal (see, for example, Meacham 1992, Miyazaki, Ueno and Shimonishi 1999, Dritschel, Scott and Reinaud 2005). Such vortices may be unstable when elongated in one direction but a spherical vortex (in a reference frame stretched by $N / f$ in the vertical direction) of uniform $\mathrm{PV}$ is neutrally stable. The later result follows from Dritschel (1988). Results have been more recently extended beyond the quasi-geostropic regime in Tsang and Dritschel (2015) to include ageostrophic effects.

Other families of vortices include shielded vortices. These vortices consist of the core of (potential) vorticity of given polarity surrounded by a distribution (or shield) of (potential) vorticity of the opposite sign. Because of cancellation due to the equal and opposite vorticity distribution in the structure, the velocity falls very rapidly away from the vortex. The vortex can be seen as generating a rather quiet external environment. The purpose of the paper is to

Corresponding author: jean.reinaud@st-andrews.ac.uk

The University of St Andrews is a charity registered in Scotland SC013532 
further explore the properties of such vortices in a theoretical and idealised set-up. Shielded structures have been extensively studied within the context of two-dimensional vortex dynamics (see, for example, Flierl 1988, Carton 1992, and Morel and Carton 1994, as well as more recently with the context of surface quasi-geostrophy by Harvey, Ambaum and Carton (2011)). The outcome is that these structures often break into secondary structures due to barotropic instabilities associated with the shear induced by the positive and negative vorticities. The instability can be violent and can result in the formation of a plethora of secondary structures, rendering a complex and rich (rather than quiet) dynamics. In these two-dimensional studies, the vortex is a circular patch of vorticity surrounded by a ring of opposite-signed vorticity. Shielded vortices have also been studied with the context of two (or three)-layer models (see, for example, Benilov 2004 and references therein). In a layered model, a shielded vortex is a cylinder of potential vorticity filling the height of a layer, surrounded by a cylindrical ring of opposite-signed PV. Again, isolated shielded vortices are in general unstable (but may be stabilised by an external flow). Most of the studies have use smooth potential vorticity distributions where the angular velocity has a Gaussian profile. Gaussian vortices were also is in three dimensional, quasi-geostrophic regime (see for example, Model and McWilliams 1997). There are experimental (van Heijst and Clercx, 2009) and observational (McWilliams, 1985 among others) evidences to support this choice of profile. It should be noted that a smooth profile is also required for Eulerian numerical simulations. These vortices appear to be unstable to small perturbations (see Sutyrin 2015 for a discussion). More recently, in the absence of background rotation, Yim and Billiant (2016) have studied the stability of a stratified vortex with a Gaussian angular velocity profile in cyclostrophic and hydrostatic balances for various values of height-to-width aspect ratio of the structure. The authors have shown that the vortex may be sensitive to centrifugal, shear, gravitational and baroclinic instabilities, depending in particular on the Reynolds and Froude numbers, and the vortex aspect ratio.

There are however theoretical and numerical evidences that at very high Reynolds number, the weak peripheral (potential) vorticity of smooth vortices can be stripped and eroded by weak external shear and straining flows (see, for example, Mariotti, Legras and Dritschel 1994). This yields to structures whose vorticity distribution exhibits sharp edges. A vortex with discontinuous, piecewise uniform, potential vorticity is a well-suited candidate for a simplified, theoretical study at the limit of infinite Reynolds number. It should be noted that such vortices are ideally suited for our Lagrangian numerical approach, based on Contour Dynamics.

More recently, Viúdez (2015) proposed steady solutions for shielded spheroidal vortices. The families of vortices obtained by the author have a continuous internal PV distribution. The motivation of the author for surrounding the vortex by a shield of opposite-signed PV is to remove a problem associated with an otherwise unbounded mass anomaly (if the mass anomaly is integrated throughout an infinite domain).

In this paper, we revisit the idea of shielding a fully three-dimensional spheroidal vortex with a spheroidal shell of opposite-signed PV. It is important to note that this case is different from the two-dimensional or the layered problem in which the vortices are only shielded laterally. For the spherical vortex, the shell is isotropic such that the core vortex is surrounded by opposite-signed PV on the bottom and on the top as well as on its sides. This adds a baroclinic component to the structure. In this work, we consider vortices where PV is piecewise uniform.

Miyazaki, Fujiwara and Yamamoto (2003) studied the linear stability of confocal, piecewise uniform potential vorticity ellipsoidal vortices. We start by revisiting these results for our specific case. We show that most of such vortices are dynamically unstable and break into secondary structures, unless the outer shield is thick, with low PV. We also briefly extend this investigation to cases of oblate and prolate spheroids, and to shielded cylindrical vortices. The latter are closer in their geometry to the cases studied in layered-models.

Finally we extend the results to the non-hydrostatic regime where both the Froude and 
Rossby numbers are finite. It should be noted that in this regime, the dynamical symmetry between cyclonic and anti-cyclonic potential vorticity (observed in the quasi-geostrophic regime) is broken. Hence, we distinguish shielded vortices with a cyclonic core and anti-cyclonic shield from their counterparts with an anti-cyclonic core and cyclonic outer shield.

The papers in organised as follows. Section 2 describes the formulation of the quasigeostrophic model and sets the general geometry of the problem. The quasi-geostrophic linear stability analysis for the shielded spheres is revisited in section 3. Their nonlinear evolutions is illustrated in section 4 . The results are extended to a family of oblate spheroids, and one of prolate spheroids in section 5. Section 6 offers some comparative results for the case of shielded cylindrical vortex. Section 7 discusses effects of periodicity when imposed on the flow. Section 8 presents the extension of the results to the non-hydrostatic regime while conclusions are drawn in section 9 .

\section{Formulation}

This study starts with the linear stability and nonlinear evolution of shielded vortices whose volume-integrated potential vorticity is zero, within the $\mathrm{QG}$ model. The quasi-geostrophic model (hereinafter referred to as QG) offers a framework ideally suited for the study of mesoscale vortices, where the combined effects of both the background planetary rotation and stable density stratification are dominant. For the present study, the model may be derived from an asymptotic expansion of the three-dimensional Boussinesq equations with respect of two small parameters, the Froude and Rossby numbers. The equation reads

$$
\frac{\partial^{2} \psi}{\partial x^{2}}+\frac{\partial^{2} \psi}{\partial y^{2}}+\frac{\partial}{\partial z}\left(\frac{f^{2}}{N^{2}} \frac{\partial \psi}{\partial z}\right)=q,
$$

where $\psi$ is the streamfunction and $q$ is the potential vorticity. $f$ is the Coriolis frequency associated with the planetary rotation while $N$ is the buoyancy (or Brunt-Vaïssälä) frequency associated with the stratification. For the sake of simplicity, we take both $f$ and $N$ constant. Then, the vertical direction $z$ can be rescaled by the constant ratio $N / f$ to $z^{*}=z N / f$. The ratio $N / f$ is typically large $(\mathrm{O}(100)$ in the oceans at midlatitudes $)$. In the reference frame $\left(x, y, z^{*}\right)$, the relation between $\psi$ and $q$ is isotropic, which motivates the search of isotropic solutions such as spherical vortices. This choice is further justified by the fact that Reinaud, Dritschel and Koudella (2003) showed that vortices in three dimensional quasi-geostrophic turbulence have a characteristic aspect ratio near to unity (they are slightly oblate with an typical aspect ratio of height to diameter $\sim 0.8$ in the vertically stretched coordinate system). In the rest of the paper, we use the stretched reference frame $\left(x, y, z^{*}\right)$. This means that the spherical vortices considered in $\left(x, y, z^{*}\right)$ are in fact pancake vortices in the physical space $(x, y, z)$, as expected.

In absence of diabatic and viscous effects, the potential vorticity in (1) is materially conserved,

$$
\frac{\mathrm{D} q}{\mathrm{D} t}=0,
$$

where $\mathrm{D} \cdot / \mathrm{D} t=\partial \cdot / \partial t+u \partial \cdot / \partial x+v \partial \cdot / \partial y$ is the material derivative, in which the advecting (horizontal) velocities are

$$
u=-\frac{\partial \psi}{\partial y} \quad v=\frac{\partial \psi}{\partial x} .
$$

Although the vertical velocity is, strictly speaking, not zero in QG, it is too small to contribute to advection at his order. QG is strictly valid if $F r^{2} \ll R o \ll 1$. The Froude number $F r=\left|\boldsymbol{\omega}_{h}\right| / N$ is the ratio of the horizontal vorticity to the buoyancy frequency $N$, while the 


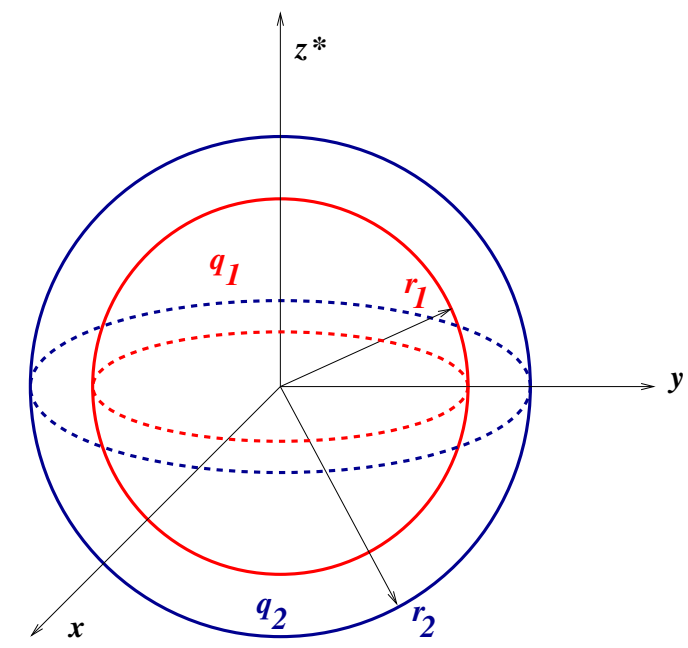

Figure 1. Geometry of the piecewise uniform shielded spherical vortex in the space $\left(x, y, z^{*}\right)$. (Colour online).

Rossby number $R o=\zeta / f$ is the ratio of the relative vertical vorticity to the background planetary vorticity $f$.

All calculations in QG regime are performed using Contour Dynamics based methods. The nonlinear simulations are performed using the fully Lagrangian Contour Surgery where the flow is explicitly unbounded (see Dritschel, 1993 for the original 2D method). The linear stability is performed by analysing deformation modes along the contours. Further details on the numerical approach may be found in Reinaud (2015) and references therein. In our calculations, the continuous vertical direction is discretised by a set of $n_{l}$ layers. The vortex boundaries are associated with potential vorticity jumps and are discretised by a set of $n_{p}$ nodes.

The geometry of the flow is presented in figure 1. The vortex core consists of a sphere is radius $r_{1}$, and of uniform PV $q_{1}$. It is surrounded by a spherical shield for $r_{1}<r<r_{2}$ of PV $q_{2}$. The vortex outer shell is such that the volume-integrated PV is zero:

$$
q_{1} r_{1}^{3}+q_{2}\left(r_{2}^{3}-r_{1}^{3}\right)=0
$$

Within the context of the Contour approach, we define two spherical surfaces. One at $r=r_{1}$ with an associated PV jump of $\delta q_{1}$, and a second spherical surface at $r=r_{2}$ corresponding to a PV jump of $\delta q_{2}$. We then have $q_{2}=\delta q_{2}$, and $q_{1}=\delta q_{1}+\delta q_{2}$. Equation (4) implies that $\delta q_{1} r_{1}^{3}=-\delta q_{2} r_{2}^{3}$. In practice, we set $\delta q_{1}=4 \pi, r_{1}=1$. We then vary $r_{2}$, adapting $\delta q_{2}$ accordingly.

\section{Linear stability of shielded PV spheres}

Miyazaki, Fujiwara and Yamamoto (2003) addressed the linear stability quasi-geostrophic confocal spheroidal vortices decomposing of the modes of instability in spherical harmonics. 
In the special case where the vortex is spherical, the dispersion relation reads ${ }^{1}$

$$
\begin{aligned}
\left(\frac{\omega_{n}^{m}}{m}\right)^{2} & -\frac{1}{3 r_{2}^{2}}\left[\frac{4 n-1}{2 n+1} r_{2}^{3} q_{1}+\left(r_{2}^{3}-r_{1}^{3}\right)\left(q_{2}-q_{1}\right)\right]\left(\frac{\omega_{n}^{m}}{m}\right) \\
& +\frac{1}{9 r_{2}^{3}}\left[\frac{2 n}{2 n+1}\left(r_{2}^{3}-r_{1}^{3}\right) q_{1}\left(q_{2}-q_{1}\right)+2 \frac{n r_{2}^{3}-r_{1}^{3}}{2 n+1} q_{1}^{2}+\frac{r_{2}^{3}-r_{1}^{3}}{2 n+1} q_{2}\left(3 q_{2}-5 q_{1}\right)\right] \\
& -\frac{1}{(2 n+1)^{2}}\left[1-\left(\frac{r_{1}}{r_{2}}\right)^{2 n+1}\right] q_{2}\left(q_{2}-q_{1}\right)=0,
\end{aligned}
$$

for each Legendre mode $\mathrm{P}_{n}^{m}(z / r) e^{\mathrm{i}\left(m \phi-\omega_{n}^{m} t\right)}$, where $\mathrm{P}_{n}^{m}(0 \leq m \leq n)$ is the associated Legendre function and $\phi$ the (horizontal) azimuthal angle (see Miyazaki et al. 2003 for details).

This dispersion relation further simplifies in our case where the integrated PV is zero. On denoting the rescaled growth rate by $\omega_{*}=\omega_{*}(n)=\omega_{n}^{m} /\left(m q_{1}\right)$, we obtain

$$
\omega_{*}^{2}-\frac{2}{3} \frac{n-1}{2 n+1} \omega_{*}+\frac{\rho_{r}^{3}}{(2 n+1)\left(1-\rho_{r}^{3}\right)}\left(\frac{1}{3}-\frac{1-\rho_{r}^{2 n+1}}{\left.(2 n+1)\left(1-\rho_{r}^{3}\right)\right)}\right)=0,
$$

where $\rho_{q} \equiv r_{1} / r_{2}$. Hence the growth rate of the unstable modes (imaginary part of $\omega_{*}$ ) is given by

$$
\gamma_{*}(n)=\left\{\begin{aligned}
\sqrt{S}, & \text { if } \quad S \geq 0, \\
0, & \text { if } \quad S<0,
\end{aligned}\right.
$$

where

$$
S=\frac{\rho_{r}^{3}}{(2 n+1)\left(1-\rho_{r}^{3}\right)}\left(\frac{1}{3}-\frac{1-\rho_{r}^{2 n+1}}{\left.(2 n+1)\left(1-\rho_{r}^{3}\right)\right)}\right)-\frac{1}{9}\left(\frac{n-1}{2 n+1}\right)^{2} .
$$

Note that when $r_{2} \rightarrow r_{1}$, i.e. $\rho_{r} \rightarrow 1$, and the inner vortex is covered by a sheet of opposite-sign $\mathrm{PV}$, the growth rates $\sigma$ have a finite limit, and

$$
\gamma_{*} \rightarrow \frac{\sqrt{(n-1)(n+2)}}{3(2 n+1)} .
$$

As mentioned by Miyazaki et al. (2003), and since the growth rate $\omega_{n}^{m}$ is proportional to $m$, for each value of $n$ the maximum growth rate is obtained for $m=n$.

These results are compared to those obtained from a general method based on the analysis of the deformation modes of the PV jumps defining the vortex. The later method has been used in three dimension in various contexts (see Reinaud and Dritschel 2002, Reinaud and Dritschel 2009, Carton and Reinaud 2009, Reinaud 2015, and Reinaud and Carton 2015). For each calculation, the core vortex is discretised by 55 layers. Each PV jump is discretised by $n_{p}=160$ nodes. The number of layers needed to discretise the outer shield depends on $r_{2}$. As for the inner PV jumps, each contour of the outer shield is also discretised by $n_{p}=160$. The choice of a constant number of nodes for each contour makes the linear stability easier, and $n_{p}=160$ guarantees an accurate representation of even the largest contours, with a horizontal resolution consistent with the vertical resolution $\left(n_{p} \sim \pi * n_{l}\right.$, where $n_{l}$ is the number of layers spanning the full structure). We study spherical shielded vortices with outer radius $r_{1}+d r<r_{2}<2 r_{1}$, with an increment of $d r=0.0025$. We additionally perform the analysis in the case $q=1=-q_{2}$, corresponding to $\delta q_{1}=4 \pi, \delta q_{2}=-\pi$ and $r_{2}=\sqrt[3]{2}$.

Each horizontal contour $k$ at height $z_{k}$ may be deformed by $M=10$ azimuthal modes

$$
r_{k}(\phi, t)=\bar{r}_{k}+\eta_{k}(\phi, t)
$$

\footnotetext{
${ }^{1}$ Note that the formula written in the original paper by Miyazaki et al. (2003) contained a typographical error, confirmed by the author (private communication).
} 

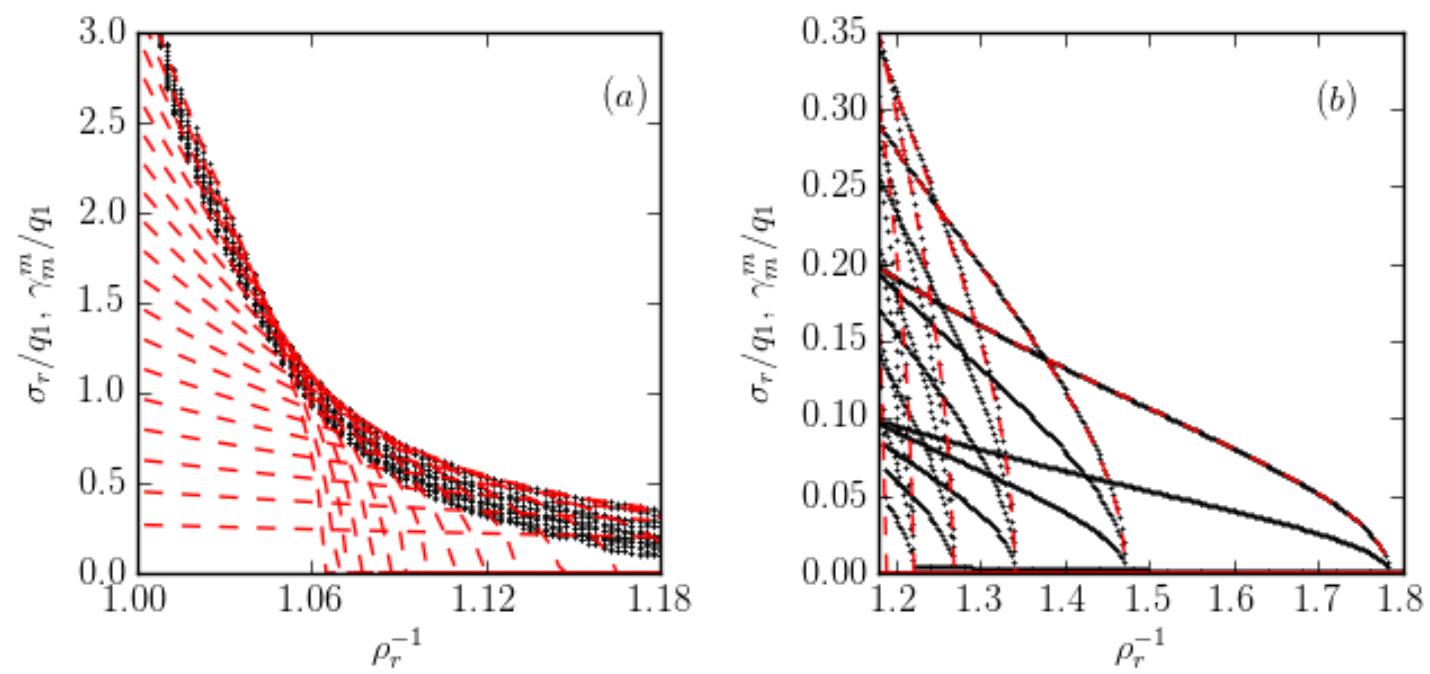

Figure 2. 40 largest growth rates for the instability modes $\sigma_{r}$ (black dots) and the corresponding 20 largest modes $\gamma_{n}^{n}$ (red dashed lines), the imaginary part of $\omega_{n}^{n}$ (using formula derived from Miyazaki et al., 2003) of a piecewise uniform, shielded spherical vortex vs. $\rho_{r}^{-1}=r_{2} / r_{1}$ the ratio of the radii of the outer shield to the inner core vortex. To ease readability we split the result for $(a): 1 \leq \rho_{r}^{-1} \leq 1.18$, and $(b): 1.18 \leq \rho_{r}^{-1} \leq 1.8$. Note that the modes $\gamma_{n}^{m}$ for $m<n$ are not shown, but are seen from $\sigma_{r}$. (Colour online).

$$
\eta_{k}(\phi)=\mathrm{e}^{\sigma t} \sum_{m=1}^{M}\left[a_{m, k} \cos (m \phi)+b_{m, k} \sin (m \phi)\right],
$$

where $r_{k}$ is the polar radial coordinate along the contour, $\bar{r}_{k}$ is the local radius of the undisturbed circular contour, and $\eta_{k}$ the perturbation. For the $n_{c}$ contours discretising the vortices, the problem results in a $2 M n_{c}$ eigenvalue problem. The eigenvalues are $\sigma=\sigma_{r}+\mathrm{i} \sigma_{i}$, where the real part $\sigma_{r}$ of the eigenmodes is the growth rate, and $\sigma_{i}$ its frequency. The eigenvectors $\hat{a}_{m, k}, \hat{b}_{m, k}$ allow to reconstruct the spatial structure of the deformation mode.

The 40 largest non-dimensional growth rates $\sigma_{r} / q_{1}$ from our analysis as a function of $r_{2} / r_{1}=$ $\rho_{r}^{-1}$ are presented in figure 2 . The figure shows a very large number of unstable modes. These modes have non-zero frequencies as well (not shown). The instabilities are favoured by small values of $r_{2} / r_{1}$, corresponding to a thin, intense outer shell. The instability modes come in pairs of complex conjugates. That is, if $\sigma=\sigma_{r}+\mathrm{i} \sigma_{i}$ is an eigenvalue, so is its conjugate $\bar{\sigma}=\sigma_{r}-\mathrm{i} \sigma_{i}$. Since these two modes are similar, we will not distinguish them in the discussion. Additionally, if $\sigma$ is a mode, so is $-\sigma$ (any unstable mode pairs with a stable counterpart). This means that each mode appears as a quartet, $\sigma,-\sigma, \bar{\sigma},-\bar{\sigma}$. For comparison, we also plot the maximum modes $\gamma_{m}^{m} / q_{1}$, obtained from the formula derived from Miyazaki et al. (2003). We see that they coincide with the largest modes $\sigma_{r}$. To ease readability, we do not include the modes $\gamma_{n}^{m}$ for $m<n$. Recall that such modes can be deduced from $\omega_{n}^{n}$ by simple scaling as $\omega_{*}(n)=\omega_{n}^{m} / m=\omega_{n}^{n} / n$. These modes are clearly captured by our calculation.

The spatial structure of the deformation for the first three most unstable modes for the case $\left|q_{2} / q_{1}\right|=1, r_{1}=1$, and $r_{2}=\sqrt[3]{2}$ are represented in figure 3 . The contours of iso-value of the deformation magnitude are drawn in a $(\phi, z)$ coordinate system on the surface of the inner vortex. The deformation of the outer shell follows a similar pattern. We see that the most unstable mode corresponds to an azimuthal wave number $m=3$, while the second has $m=4$ and the third $m=2$. However, the growth rates have similar order of magnitude for the three, with $\sigma(m=3) \simeq 0.229 q_{1}, \sigma(m=4) \simeq 0.222 q_{1}$, and $\sigma(m=2) \simeq 0.172 q_{1}$.

In our calculation, unstable modes disappear for $r_{2} / r_{1}$ larger than a threshold $1.7825 \leq$ $\left(\rho_{r}^{-1}\right)_{m} \leq 1.785$. Using $n=2$ (the Legendre mode unstable for the largest range of the 

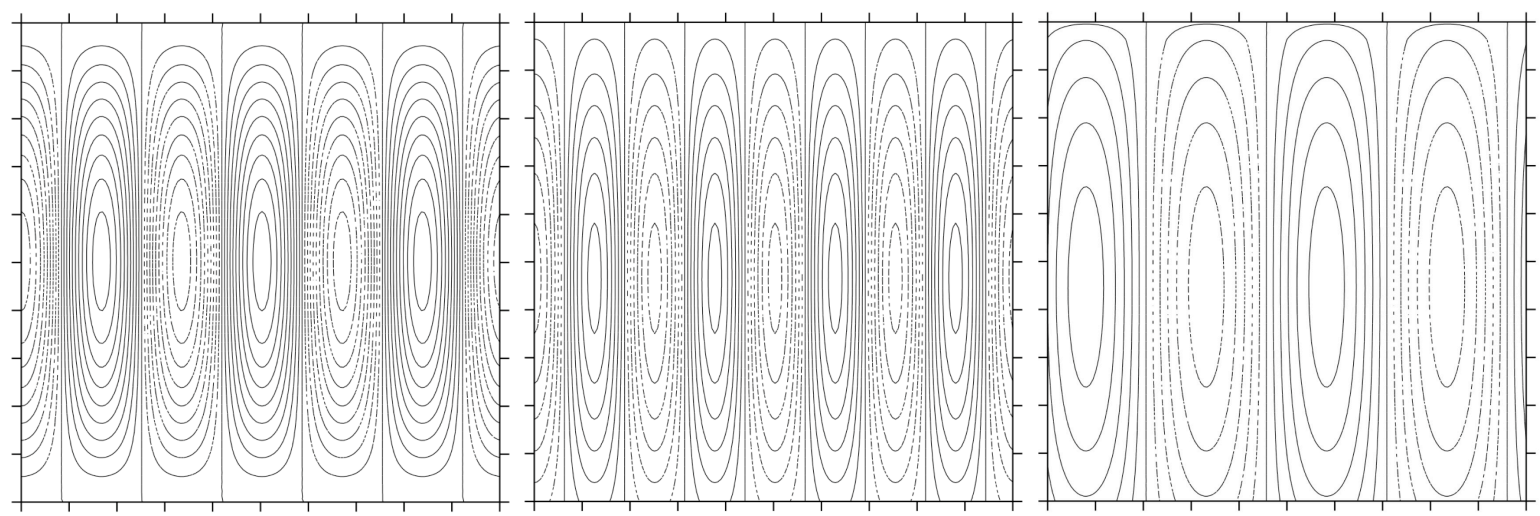

Figure 3. Reconstructed deformation mode on the boundary of the inner vortex for the first three most unstable modes (ordered from left to right from most to least) for the piecewise uniform shielded vortex, with $\left|q_{2} / q_{1}\right|=1, r_{1}=1$, and $r_{2}=\sqrt[3]{2}$. The vertical axis of each frame corresponds to the vertical coordinate $z$ along the inner vortex surface, and the horizontal axis is the azimuthal angle $\phi$ around the vortex surface. The lines correspond to contours of iso-value of deformation amplitude.
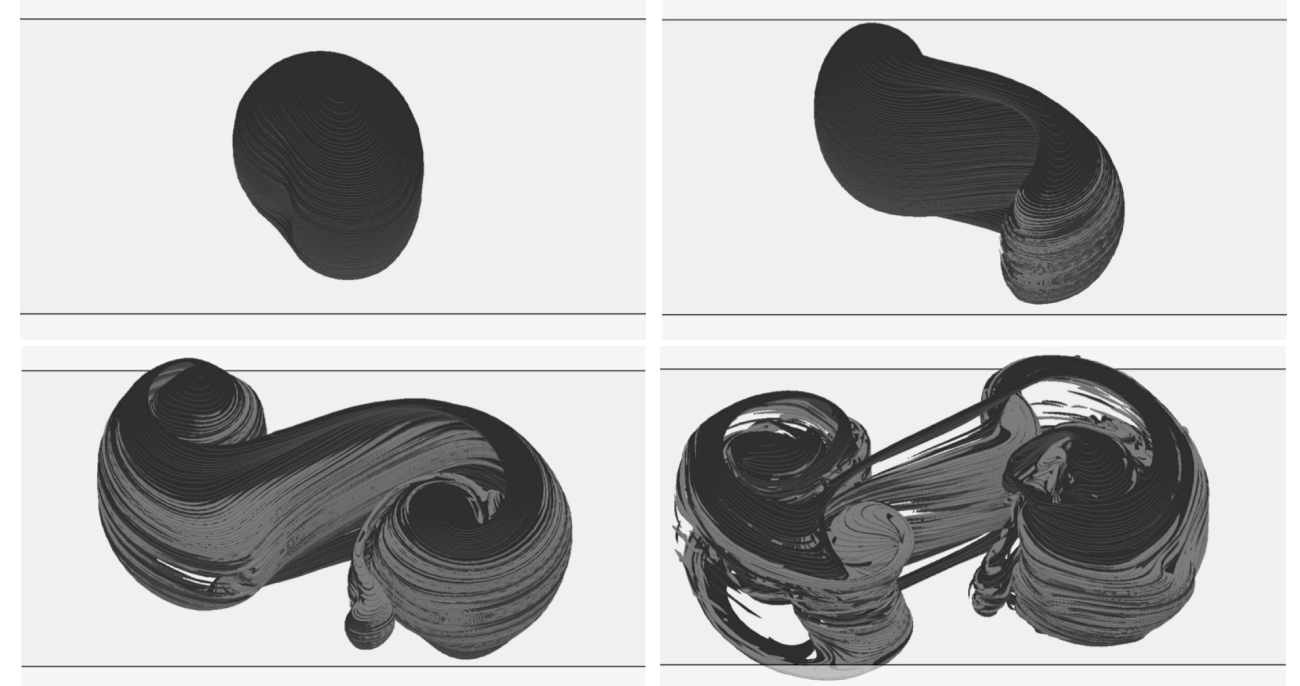

Figure 4. PV contours defining the vortex boundary. The outer shell is dark grey, and the contours associated with the internal core are lighter grey. The view is seen orthographically at an angle of $60^{\circ}$ from the vertical. Time displayed at $t=4.5,7,11.5,16$. (Colour online).

parameter $\rho_{r}$ ), and solving for $S=0$ in equation (8), we obtain $\rho_{r}=0.5604256605$ which corresponds to $\left(\rho_{r}^{-1}\right)_{m} \simeq 1.7844$, confirming the accuracy of our calculation.

\section{Nonlinear evolution of shielded PV spheres}

We next illustrate the nonlinear evolution of the piecewise uniform shielded spherical vortices. This is done using the purely Lagrangian method of Contour Dynamics. This means that the flow is explicitly unbounded with $\psi, \boldsymbol{u} \rightarrow 0$ when $|\boldsymbol{x}| \rightarrow \infty$. In each case, the flow is mapped in the vertical by $n_{l}=100$ layers from the bottom to the top of the outer shield. In all cases we set $\delta q_{1}=4 \pi$, and $r_{1}=1$. Recall that $\delta q_{2}=-\delta q_{1}\left(r_{1}^{3} / r_{2}^{3}\right)$. The PV of the inner vortex is therefore $q_{1}=\delta q_{1}+\delta q_{2}$. The time scale of the problem is set by PV. Recall that a sphere of uniform PV $q$ has a rotation period of $T=6 \pi / q$.

Figure 4 illustrates the time evolution of a spherical shielded vortex for $r_{2}=\sqrt[3]{2}$. This case corresponds to the case where the inner core and the shell have equal intensities $q_{1}=-q_{2}$. 

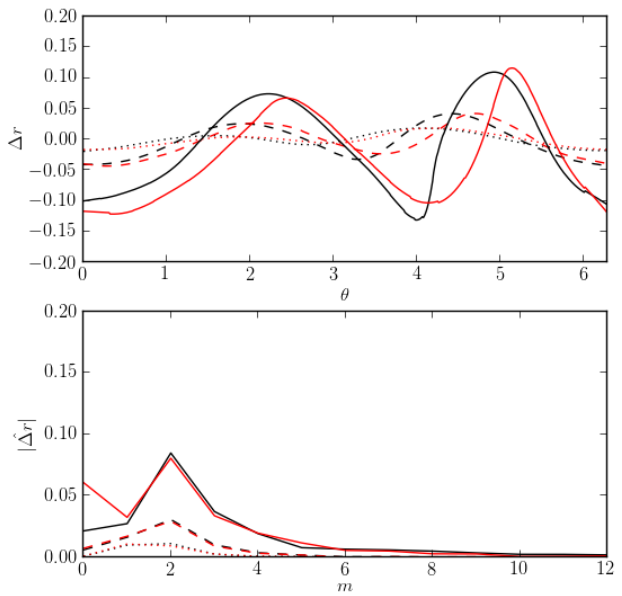

Figure 5. Deformation of the PV jumps defining the horizontal mid-section of the shielded vortex with $r_{2} / r_{1}=\sqrt[3]{2}$, $\left|q_{2} / q_{1}\right|=1$ at $t=3$ (dotted line) 4 (dashed line) and 5 (solid line). Top: deformation in physical space $\Delta r$ vs. the local polar angle $\theta=\phi-\phi_{0}^{i}$ where $\phi_{0}^{i}$ is the polar angle of the first node for the contour $i$. Bottom: Amplitude of the Fourier modes $|\hat{\Delta r}|$ vs. the azimuthal wave number $m$ (only small $m$ shown). (Colour online).

Its linear stability is addressed in figures 2 and 3. We do not introduce a prescribed initial disturbance on the vortex. The instabilities are excited by the low amplitude background noise inherent to any discretised numerical approach. The nonlinear evolution shows the complex growth in interaction of many modes of instabilities, in particular the modes $m=3,4$, and 2 mentioned in the previous section. Possibly because of coupling between modes $m=4$ and $m=2$, the structure mostly separates as two structures. This break-up is asymmetric due to the influence of mode $m=3$. The mode $m=3$ is most visible at the early stages of the evolution (see figure 4, top left at $t=4.5$ ), which is consistent with the linear stability results. The later, nonlinear evolution is much more complex due to the nonlinear interaction between the numerous modes which have different symmetries. Nevertheless, it is clear that the vortex is strongly unstable, and breaks into secondary structures. The evolution of the instability modes can be monitored at early stages. We start by extracting the inner and outer contours at the horizontal mid-section of the structure. We then calculate $\Delta r$ the departure of the local radius to the mean radius along the contour. This signal is then re-sampled on 256 nodes equally spaced in polar angle $\theta=\phi-\phi_{0}^{i}$ using local quadratic interpolation. The angle $\phi_{0}^{i}$ corresponds to the polar angle of the first node of the contour. The latter distribution is analysed in Fourier space. Results at $t=2,3$ and 5 are presented in figure 5 . The early nonlinear evolution is dominated by the mode $m=2$ (due to nonlinear coupling of the modes) but modes $m=3$ and $m=4$ are also developing. The small amplitude mode $m=1$ observable corresponds to a shift of the structure due to nonlinear effects.

We next illustrate a case where $\left|q_{2}\right|>q_{1}$ with $r_{2}=1$.1. In this case $q_{2} \simeq-3 q_{1}$. Note that this case corresponds to the part of the parameter space where many modes are unstable. We therefore expect the vortex evolution to be more complex (and to depart from equilibrium rapidly). The flow is illustrated in figure 6 . Due to the large number of competing, strongly unstable modes are developing and interacting at the same time. The spatial structure of the deformation is highly complex and cannot be simply linked to a single dominant mode. The flow develops a turbulent-like behaviour very rapidly. As done in the previous case, we analyse at early stage the deformation modes of the PV jumps at the horizontal mid-section of the vortex. Note that the analysis can only be performed meaningfully provided the signal $\Delta r$ vs. the local polar angle is a single-valued function. The approach is no longer valid when the contours overturn (which occurs in this case as early as $t \simeq 3.5$ ). Results at $t=3$ are shown in 

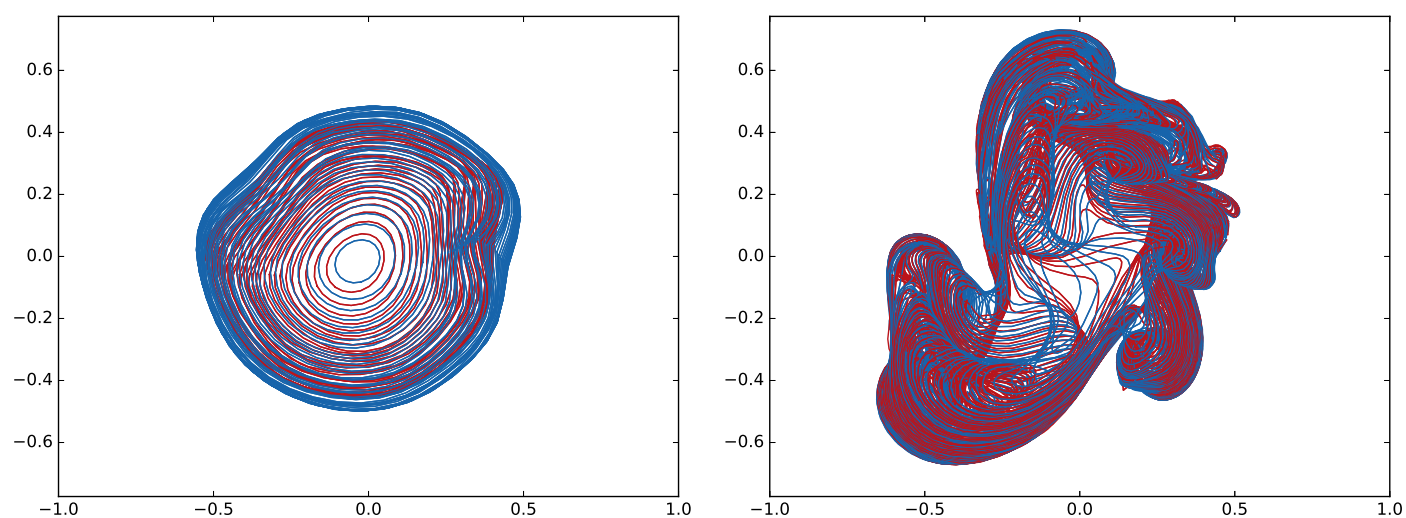

Figure 6. Top view of the PV contours for a piecewise uniform spherical vortex with a thin, intense, shield. Blue contours correspond to the negative PV jumps of the initial outer shell while the red contours indicate positive PV jumps of the inner core. The case presented corresponds to $r_{2}=1.1$, with $q_{2} \simeq-3 q_{1}$. Left: $t=3.5$, right: $t=7.5$. (Colour online).

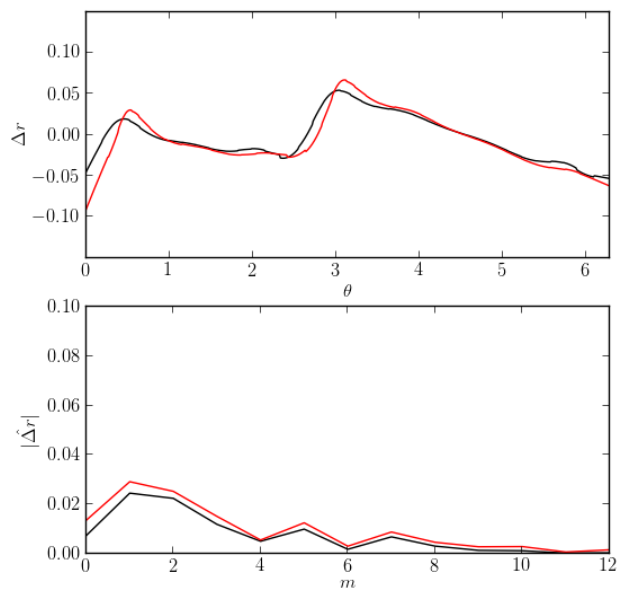

Figure 7. Deformation of the PV jumps defining the horizontal mid-section of the shielded vortex with $r_{2} / r_{1}=1.1$, $\left|q_{2} / q_{1}\right| \simeq 3$ at $t=3$. Top: deformation in physical space $\Delta r$ vs. the local polar angle $\theta$, bottom: Amplitude of the Fourier modes $|\hat{\Delta r}|$ vs. the azimuthal wave number $m$ (only small $m$ shown). (Colour online).

figure 7 . The results show that even at early stage, many modes of instability, with azimuthal wave numbers up to at least 10, are developing simultaneously.

Next, we illustrate an example where $r_{2} / r_{1}=1.6$. In this case, $\left|q_{1}\right|>\left|q_{2}\right|$ with $q_{2} \simeq-q_{1} / 3$ in figure 8. This corresponds to a thicker shield of weaker PV. The volume of the spherical shield is three times the volume of the core of the vortex. Even in this case, the structure is strongly unstable, albeit to fewer modes of instability compared to the two previous examples. The spatial structure of the vortex is less complex as it evolves in time. Nonetheless, the instability leads to the break-up of the vortex. The main mode of instability has a azimuthal wave number $m=2$. This is confirmed by the analysis of the contour deformation at early times presented in figure 9, where we clearly see the dominance of the mode $m=2$. The main structure is first elongated (in a way consistent with the mode $m=2$ ) until it breaks into two main parts together with small filaments and PV debris (late evolution not shown). For each main structure formed, a part of the peripheral PV reorganises itself as a pole on the side the core. The core itself is now only shielded by a thinner layer of opposite-signed PV, hence is globally 'signed' as the shield no longer compensates its core PV. Each structure resembles a dipole which translates away form the centre of the domain. Note that a similar late behaviour is observed for $r_{2} / r_{1}=\sqrt[3]{2}$, however the formation of the dipole-like structures 

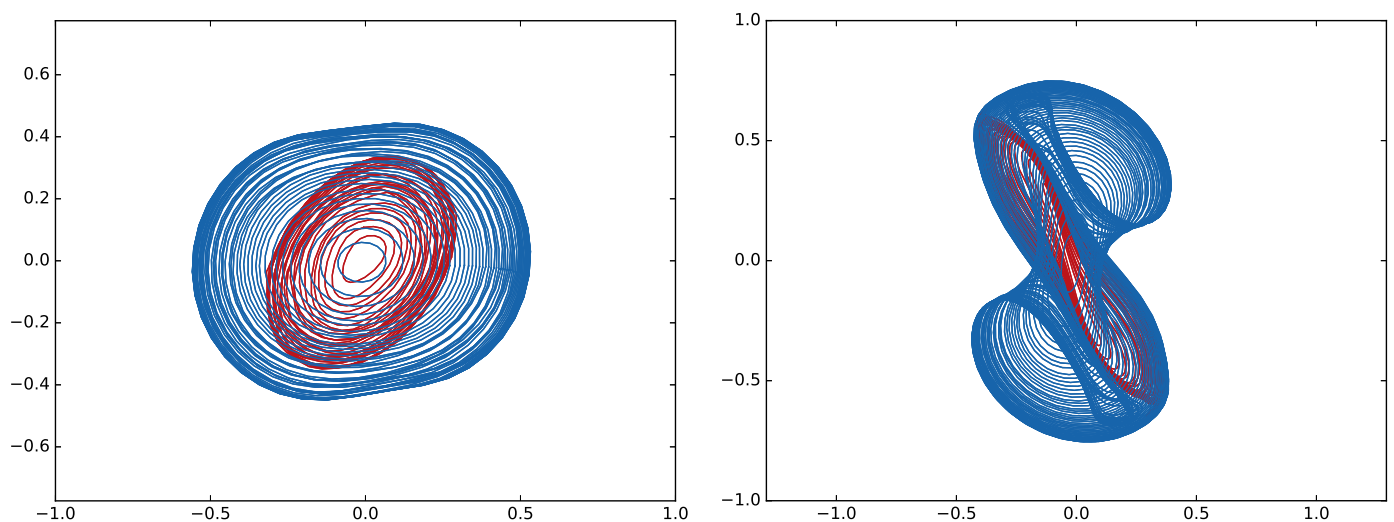

Figure 8. Top view of the PV contours for a piecewise uniform spherical vortex with a medium-thickness, weak PV, shield. Blue contours corresponds to the negative PV jumps of the initial outer shell while the red contours indicate positive PV jumps of the inner core. The case presented corresponds to $r_{2}=1.6$, with $q_{2} \simeq-q_{1} / 3$. Left: $t=8$, right: $t=11$. (Colour online).
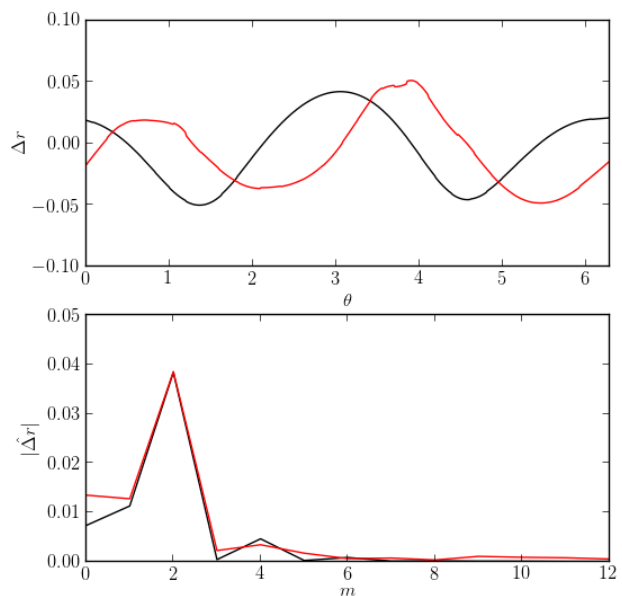

Figure 9. Deformation of the PV jumps defining the horizontal mid-section of the shielded vortex with $r_{2} / r_{1}=1.6$, $\left|q_{2} / q_{1}\right|=\simeq 1 / 3$ at $t=7.5$. Top: deformation in physical space $\Delta r$ vs. the local polar angle $\theta$, bottom: Amplitude of the Fourier modes $|\hat{\Delta r}|$ vs. the azimuthal wave number $m$ (only small $m$ shown). (Colour online).

is less clear as more modes of instability are competing in the evolution of the flow. In the former case with $r_{2} / r_{1}=1.1$, the structure becomes extremely complex very rapidly, limiting the re-organisation of the structure into dipoles. Indeed instabilities with higher wave numbers tend to generate sub-structures at finer scales.

\section{Extension to spheroids}

We now briefly extend our results to two classes of spheroidal vortices of aspect ratio $\alpha=h / r$, where $h$ is the half-height of the structure and $r$ is their mean horizontal radius. We show that the overall behaviour observed in the previous section is generic and not specific to the spherical geometry. We impose $\alpha=h_{1} / r_{1}=h_{2} / r_{2}$ for the sake of simplicity (i.e. the core and the shield have the same aspect ratio). The volume on the core is therefore $V_{1}=4 \pi r_{1}^{2} h_{1} / 3=$ $4 \pi \alpha r_{1}^{3} / 3$ while the outer shield has a volume $V_{2}=4 \pi \alpha\left(r_{2}^{3}-r_{1}^{3}\right) / 3$. Therefore the volume ratio is $V_{2} / V_{1}=\left(r_{2} / r_{1}\right)^{3}-1$ as for the spherical case. The only difference with the spherical case is that we either squash $(h / r<1)$ or stretch $(h / r>1)$ the vertical axis of the original spherical vortex. The growth rates for $h / r=0.5$ and $h / r=2$ vs. $r_{2} / r_{1}$ are presented in figure 10 . 

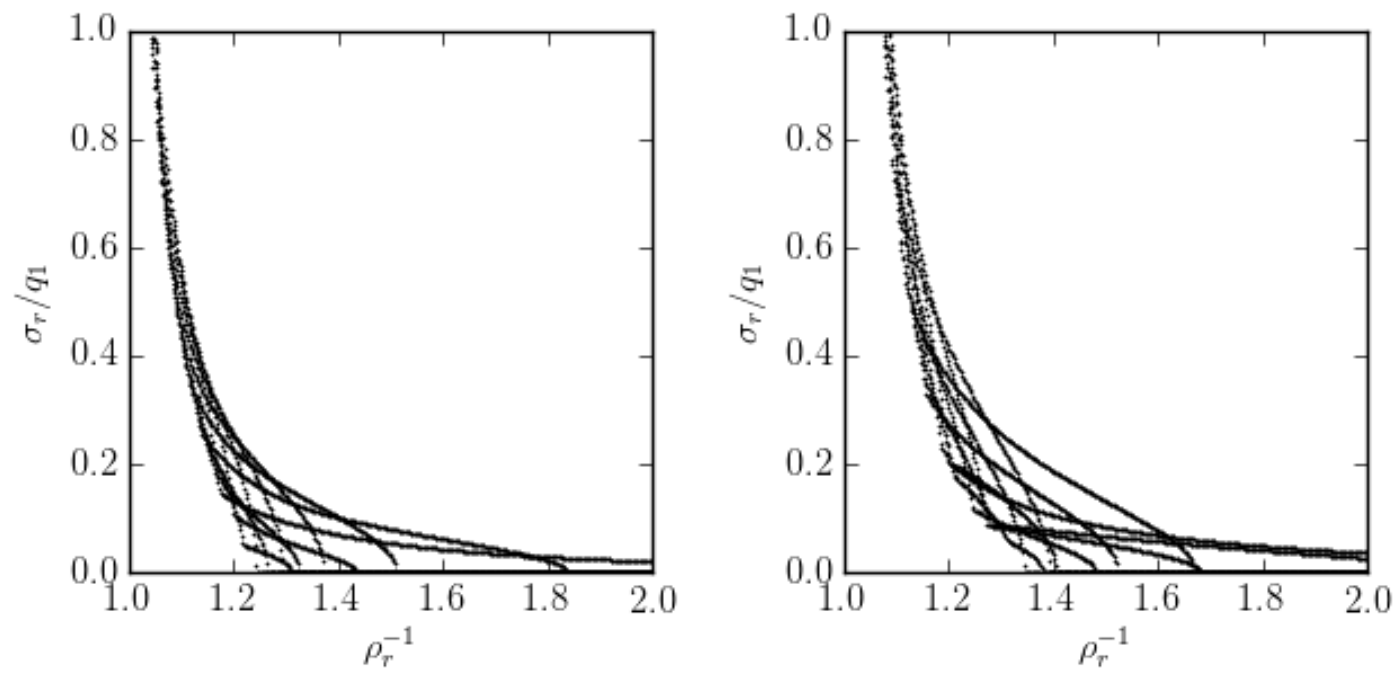

Figure 10. 10 largest non dimensional growth rates $\sigma_{r} / q_{1}$ for the instability modes of a piecewise uniform, shielded spheroidal vortices vs. $\rho_{r}^{-1}=r_{2} / r_{1}$ the ratio of the radii of the outer shell to the inner core vortex. Left: $h / r=0.5$, right: $h / r=2$.

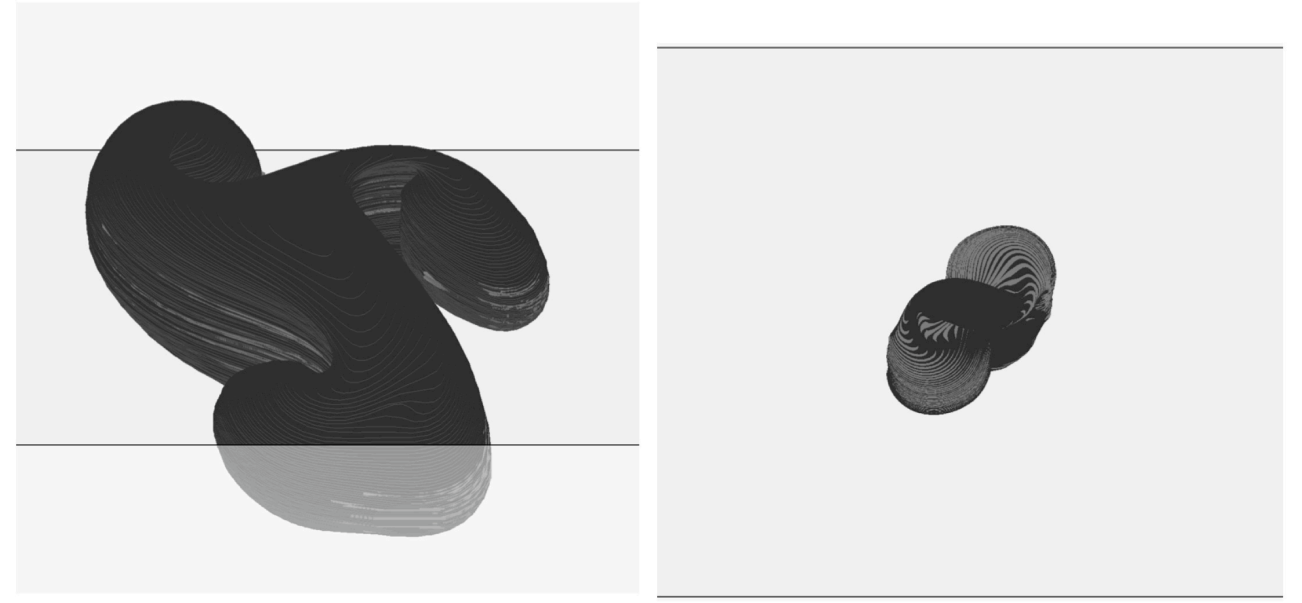

Figure 11. PV contours defining the vortex boundary for $\left|q_{2} / q_{1}\right|=1, r_{2}=\sqrt[3]{2} r_{1}$ at $t=7.5$. Left: $h_{1} / r_{1}=h_{2} / r_{2}=0.5$, orthographic view at $45^{\circ}$ from the vertical. Right: $h_{1} / r_{1}=h_{2} / r_{2}=2$, orthographic view at $15^{\circ}$ from the vertical.

The overall situation is similar, with strong instabilities associated with small values of $r_{2}$. The smaller the aspect ratio $h / r$ is, the wider the range $r_{2}$ for instability is. The weak, slowly decaying main modes which are still unstable at large values of $r_{2}$ are non-destructive modes with an azimuthal wave number $m=1$. In the case of $h / r=2$ though, there is still a secondary unstable mode with $m=2$. This mode is destructive, and is illustrated in the appendix $\mathrm{A}$ for $r_{2} / r_{1}=2$.

We next illustrate the nonlinear evolution of the spheroidal shielded vortices when they are unstable. In the first example, we set $r_{2}=\sqrt[3]{2} r_{1}$ such that $q_{1}=-q_{2}$. We consider two cases, one with $h / r=0.5$, and one with $h / r=2$. Results are presented in figure 11 . The main influence of the aspect ratio is seen to be the preferred (or most amplified) mode. The dominant mode for $h / r=0.5$ is $m=3$. The vortex eventually breaks asymmetrically into three compound structures. Again, the asymmetry is the result of the influence of other modes. For $h / r=2$, the structure breaks into two main structures due to the preponderance of the mode $m=2$. Again in this case, for each secondary structure created, the centre of PV of 


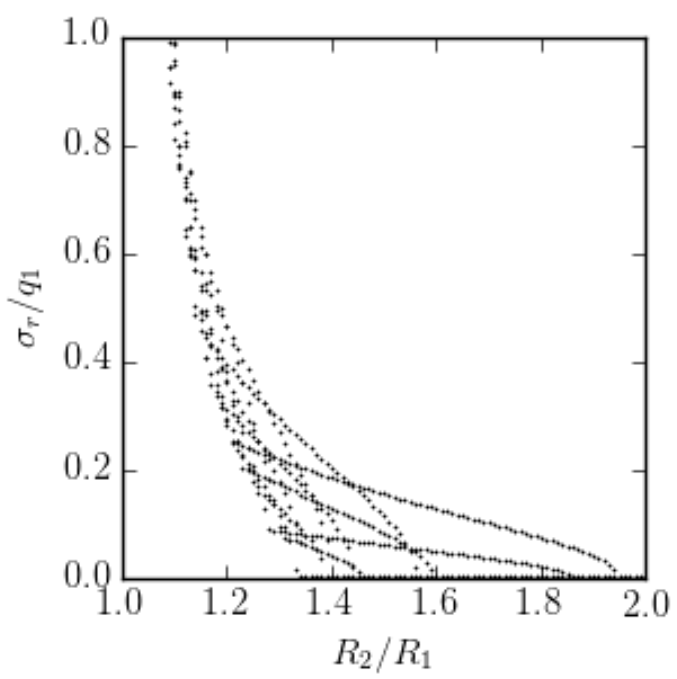

Figure 12. 10 largest growth rates for the instability modes of a piecewise uniform, laterally shielded cylindrical vortex (with $h_{1} / R_{1}=1$ ) vs. $R_{2} / R_{1}$ the ratio of the horizontal radii of the outer shell to the inner core vortex.

the positively signed-PV and negatively signed PV are offset, creating a dipolar effect which allows the structures to move away from the centre of the domain (and away from each other).

\section{Case of laterally shielded PV cylinders}

We next confirm that the cylindrical shielded vortices have similar properties. This is of course expected from the known results in two-dimensional and shallow water models. The instability of such configurations is, for example, addressed in Flierl (1988) in shallow water, and by Carton and Legras (1994) in two dimensions. In order to remain close to the spherical vortices, we consider a cylindrical inner core of aspect ratio $h_{1} / R_{1}=1$, where $h_{1}$ is the half height of the cylinder and $R_{1}$ is its horizontal radius of the inner core. The outer shield has the same height $h_{2}=h_{1}$ and has a radius $R_{2}$. Again we impose that the volume-integrated $\mathrm{PV}$ is zero by

$$
q_{1} R_{1}^{2}+q_{2}\left(R_{2}^{2}-R_{1}^{2}\right)=0 .
$$

In this case, this means that the surface integrated PV at each vertical level is zero. As before the vortices are defined by two PV jumps $\delta q_{1}$ and $\delta q_{2}$ such that $q_{2}=\delta q_{2}$, and $q_{1}=\delta q_{1}+\delta q_{2}$. We set $\delta q_{1}=4 \pi, h_{1}=R_{1}=1$, and vary $R_{2}$. The result of the linear stability analysis are presented in figure 12 . Overall the instability diagram exhibits the same generic pattern. For small values of $R_{2}$ (i.e. thin, intense outer shield), the structure is strongly unstable (many modes of instability with large growth rates) and the vortices become less unstable (fewer modes with smaller growth rates) as the shield is made thicker and its PV decreased.

An illustration of the flow evolution for $q_{2}=-q_{1},\left(R_{2}=\sqrt{2} R_{1}\right)$ is proposed in figure 13 . The core and the outer shield deform. The deformation is dominated by an azimuthal mode $m=2$. This is confirmed quantitively by the analysis of the deformation modes which develop in the nonlinear simulation presented in figure 14. The structure eventually breaks into two parts which move apart from one another, showing a behaviour similar to pairs of vortex dipoles. This is very similar to what is observed in two-dimensions. 

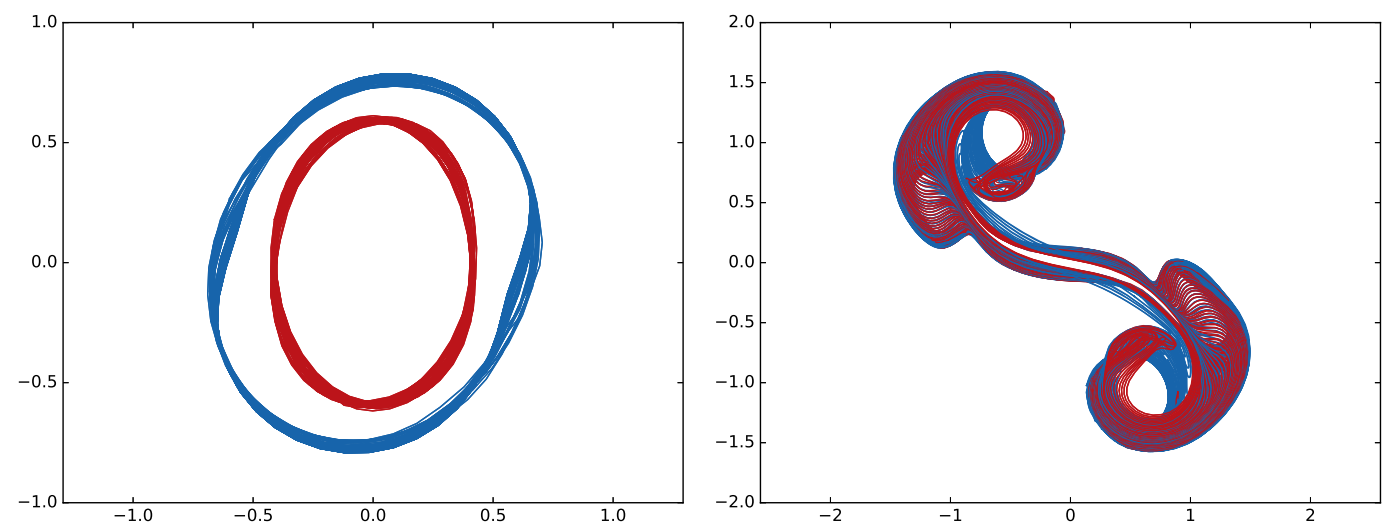

Figure 13. Top view of the PV contours for a piecewise uniform cylindrical vortex with a medium-thickness, weak PV, shield. Blue contours corresponds to the negative PV jumps of the initial outer shell while the red contours indicate positive PV jumps of the inner core. The case presented corresponds to $R_{2} / R_{1}=\sqrt{2}$, with $\left|q_{2} / q_{1}\right|=1$. Left: $t=20$, right: $t=25$. (Colour online).
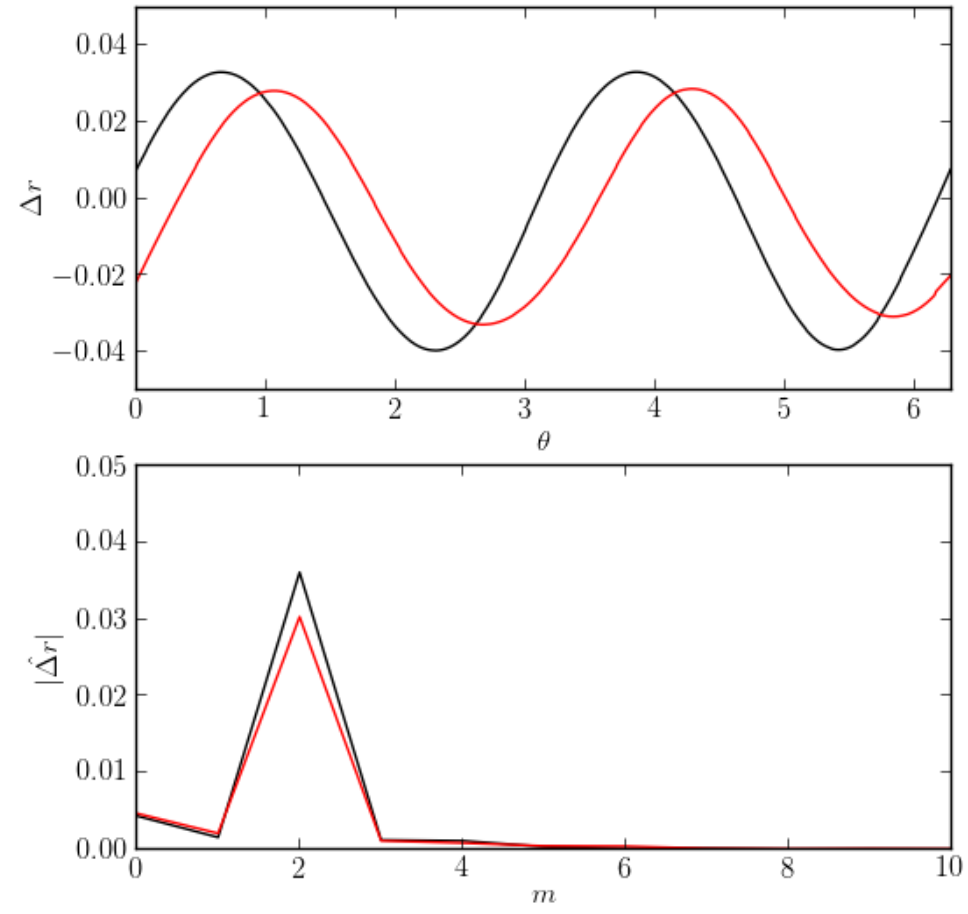

Figure 14. Deformation of the PV jumps defining the horizontal mid-section of the shielded cylindrical vortex with $R_{2} / R_{1}=\sqrt{2},\left|q_{2} / q_{1}\right|=1$ at $t=18.5$. Top: deformation in physical space $\Delta r$ vs. the local polar angle $\theta$, bottom: Amplitude of the Fourier modes $|\hat{\Delta r}|$ vs. the azimuthal wave number $m$ (only small $m$ shown). (Colour online).

\section{Periodicity}

The final part of the present research consists in a brief exploration of the effects of finite Rossby and Froude numbers on the evolution of the flow. For this, we use the numerical method developed by Dritschel and Viùdez (2003). The method is semi-Lagrangian and relies on the (spectral) inversion of the dynamical fields on a periodic grid. Before turning our attention to this problem, we illustrate the effects of periodicity in the quasi-geostrophic regime. In this regime, we can compare the results from our purely Lagrangian approach where the flow is explicitly unbounded, to the results obtained using the Contour-Advective 

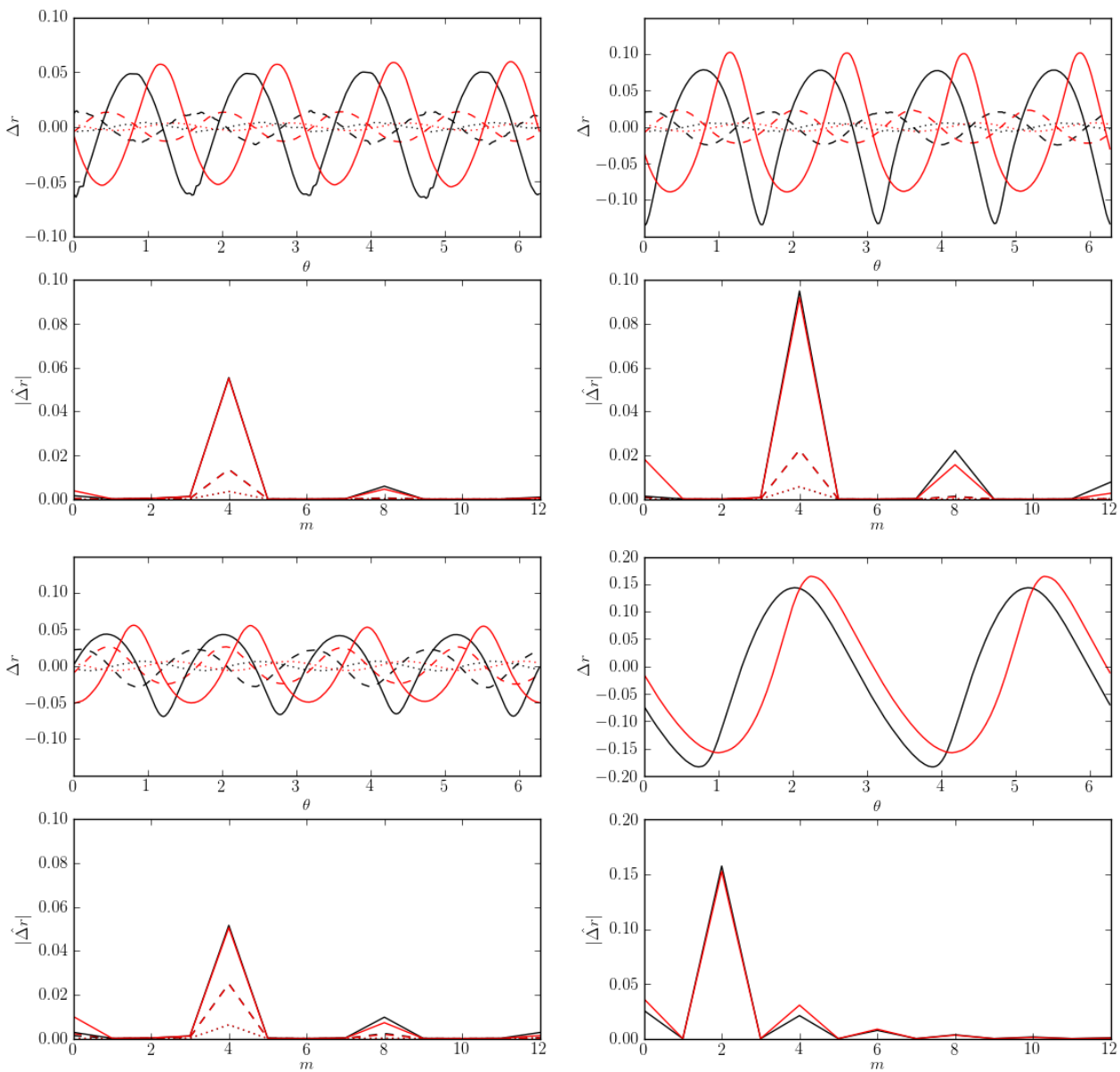

Figure 15. Deformation of the horizontal mid-section contours for CASL simulations for a spherical shielded vortex with $r_{2} / r_{1}=\sqrt[3]{2}$ and $\left|q_{2} / q_{1}\right|=1$ for $r_{1}=1$ and $t=5$ (top left), $r_{1}=0.75$ and $t-5$ (top right), $r_{1}=0.5$ and $t=4.5$ (bottom left). For $r_{1}=0.5$, at $t=5$ the contour deformation is already no longer a single-valued function of $\theta$. Bottom right: $r_{1}=0.75$ at $t=5$, with azimuthal mode $m=2$ forced initially, see details in the text. (Colour online).

Semi-Lagrangian algorithm (CASL) (see Dritschel and Ambaum (1997) for details) which imposes periodic boundary conditions.

To this purpose, we use a generic example of a spherical shielded vortex with $r_{2} / r_{1}=\sqrt[3]{2}$ and $\left|q_{2} / q_{1}\right|=1$, as used in figures 4 and 5 . We perform CASL simulations with a triply periodic inversion (coarse) grid with $256^{3}$ nodes in a physical domain $D$ of size $(2 \pi)^{3}$. The effects of the periodic images of the shielded vortex increases as the the size of the structure increases in the computational box. On the other hand, for a fixed grid resolution, the accuracy of the description of the vortex decreases as the size of the vortex is decreased (fewer grid points map the vortex). A compromise between accuracy and adverse effects of periodicity has to be made. We illustrate the results from four calculations in figures 15 and 16 for three different vortex sizes. Figure 15 shows the deformation modes on the horizontal mid-section contours at early times. Figure 16 illustrates the overall shape of the structures. They correspond to $r_{1}=1$, $r_{1}=0.75$, and $r_{1}=0.5$. Recall that this equilibrium is unstable to modes $m=3, m=4$ and $m=2$. In the unbounded calculation (see section 4 ), the nonlinear deformation of the contour at early stage was dominated by $m=2$. In the periodic calculation we do not introduce an initial perturbation on the vortex. The modes of instability are seeded by numerical noise and the geometry of the flow. We see from both figures 15 and 16 that the periodic conditions are naturally forcing the mode $m=4$. Since $m=4$ is, in the linear theory, the second most unstable mode, it dominates the early evolution of the flow. This is true for all three initial 

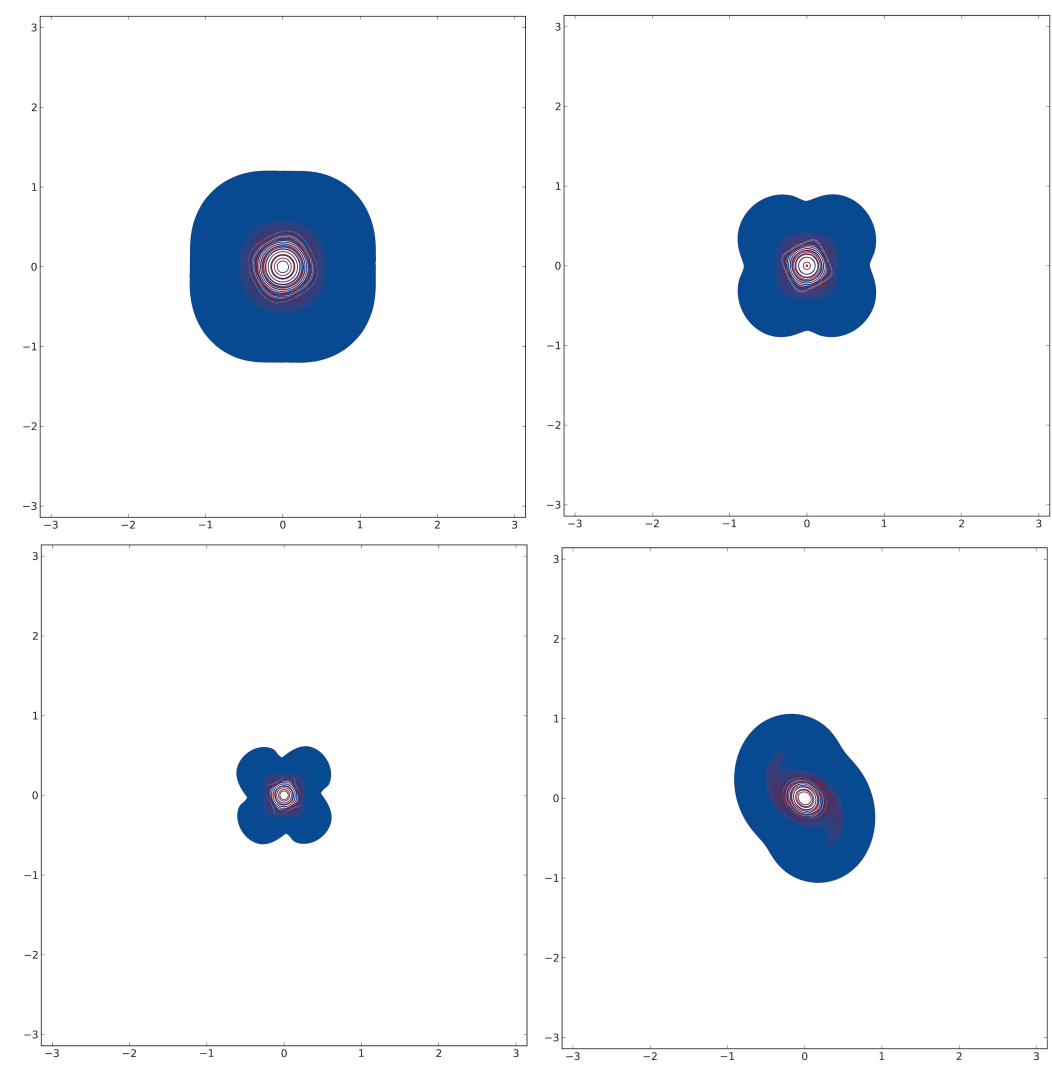

Figure 16. Top view on the vortices for CASL simulations for a spherical shielded vortex with $r_{2} / r_{1}=\sqrt[3]{2}$ and $\left|q_{2} / q_{1}\right|=1$ for $r_{1}=1$ (top left), $r_{1}=0.75$ (top right), $r_{1}=0.5$ (bottom left). Bottom right: $r_{1}=0.75$ at $t=5$, with azimuthal mode $m=2$ forced initially, see details in the text. (Colour online).

values of the radius $r_{1}$ used. The main difference between the three cases, is that the smaller the core is, the faster the deformation grows. The slowing down of the development of the modes when increasing the size of the vortex is related to a confinement effect associated with the surrounding periodic images. However, qualitatively, the three results are similar, at least at the stage of the destabilisation of the vortex, which is our main focus. We can however control the early evolution by introducing an initial perturbation to the flow. The bottom right frame of figures 15 and 16 illustrates a case (for $r_{1}=0.75$ ) where the horizontal contours are initially deformed to be elliptical. The elliptical contours have with an aspect ratio of the semi-axis lengths of 1.02 , with a phase of $\pi / 2$ between the deformation of the inner and outer contours. This is, at leading order, equivalent to forcing the azimuthal mode $m=2$. The flow then amplifies the perturbation provided as expected and the mode $m=2$ dominates the flow evolution. This shows that the underlying dynamics of the flow is not altered by the periodic conditions. In absence of explicit deformation of the initial vortex, periodicity favours the mode $m=4$ simply because it is seen as a perturbation with $m=4$ by the vortex. As a comprise between accuracy of the vortex description and effects of periodicity we use $r_{1}=0.75$ for our calculations at finite Froude and Rossby number. First, we do not introduce any additional perturbation to the initial conditions (unforced dynamics) to minimise the initial disturbances on the system. In a second part, we introduce an explicit, small yet finite amplitude, initial disturbance to promote the development of one of the unstable modes available (recall that more than one mode is linearly unstable in the QG regime). 

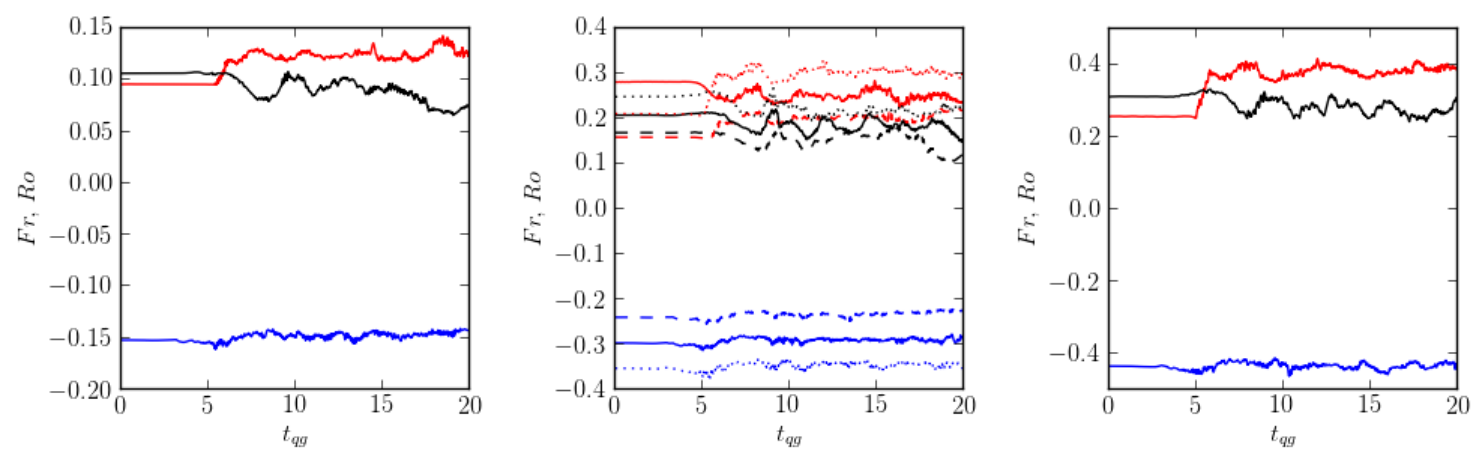

Figure 17. Non hydrostatic shielded vortex with $r_{2} / r_{1}=\sqrt[3]{2}$ and $\left|q_{1} / q_{2}\right|=1$. The vortex core is a cyclone, while the outer shield has anti-cyclonic PV. $F r^{\max }$ (black), $R o^{\min }$ (blue) and $R o^{\max }$ (red). Left: $R o_{q}=0.125$. Middle panel: $R o_{q}=0.2$ (dashed), 0.25 (solid), 0.3 (dotted). Right $R o_{q}=0.375$. (Colour online).

\section{Shielded vortices at finite Rossby and Froude numbers}

\subsection{Unforced dynamics}

We next illustrate the effect of finite Rossby and Froude numbers on the evolution of the flow. For the sake of simplicity, we focus on one geometry for the shielded vortex while varying the Rossby number. We set $r_{1}=0.75, r_{2}=\sqrt[3]{2} r_{1}$, such that $\left|q_{1} / q_{2}\right|=1$. Contrarily to the quasigeostrophic case, at finite Rossby number, there is a natural dynamical asymmetry between the cyclonic and anti-cyclonic motions.

Following the study in the QG regime, $f$ and $N$ are assumed constant, and the vertical direction is stretched by the ratio $N / f$. In our study, we set $f / N=0.1$ for convenience. It should be noted that Dritschel and McKiver (2015) showed that $f / N$ has only a weak influence on the basic properties of geophysical turbulence. We therefore expect that our specific choice of $f / N$ allows to capture the generic properties of the shielded vortex in the nonhydrostatic regime. The numerical method uses as prognostic variables Ertel's PV anomaly $q$ and ageostrophic vorticity $\boldsymbol{A}_{h}$ which is the horizontal component of the vector

$$
\boldsymbol{A} \equiv \frac{\omega}{f}+\frac{\nabla b}{f^{2}}
$$

where $\boldsymbol{\omega}=(\xi, \eta, \zeta)$ is the relative vorticity and $b$ is the buoyancy anomaly. The PV is materially conserved and is followed in a Lagrangian way, while $\boldsymbol{A}_{h}$ is represented on a Eulerian grid. These two fields can be inverted to obtain a vector potential $\boldsymbol{\psi}$ from which both $\boldsymbol{u}$ and $b$ derive. Details of the governing equations, not reproduced here, can be found in Dritschel and Viùdez (2003). As mentioned in the previous section, the inversion procedure is performed in spectral space. We used an inversion (coarse) grid of dimension $128^{3}$ mapping the triply periodic computational box $D$ of physical dimension $(2 \pi)^{3}$. The time scale $T$ of the problem is set by $N^{-1}$. We use $N=2 \pi$.

Simulations are performed at a given PV-based Rossby number $R o_{q} \equiv q_{1} / f$. The inequality $R o_{q}>0$ means that the vortex core is a cyclone while the outer shield is anti-cyclonic. The alternative inequality $R o_{q}<0$ corresponds to the reverse situation with an anti-cyclonic core and cyclonic outer shield. To control numerical stability, a bi-harmornic diffusion is applied to $\boldsymbol{A}_{h}$, where the maximum damping rate (on the highest wavenumber in spectral space) is set to $1+R o_{q}^{4}$ per inertial period (see McKiver and Dritschel 2008). The spectral fields are also de-aliased by the $2 / 3$-rule for the computation of nonlinear terms.

We define the maximum Froude number $F r^{\max }$, and minimum (resp. maximum) Rossby 

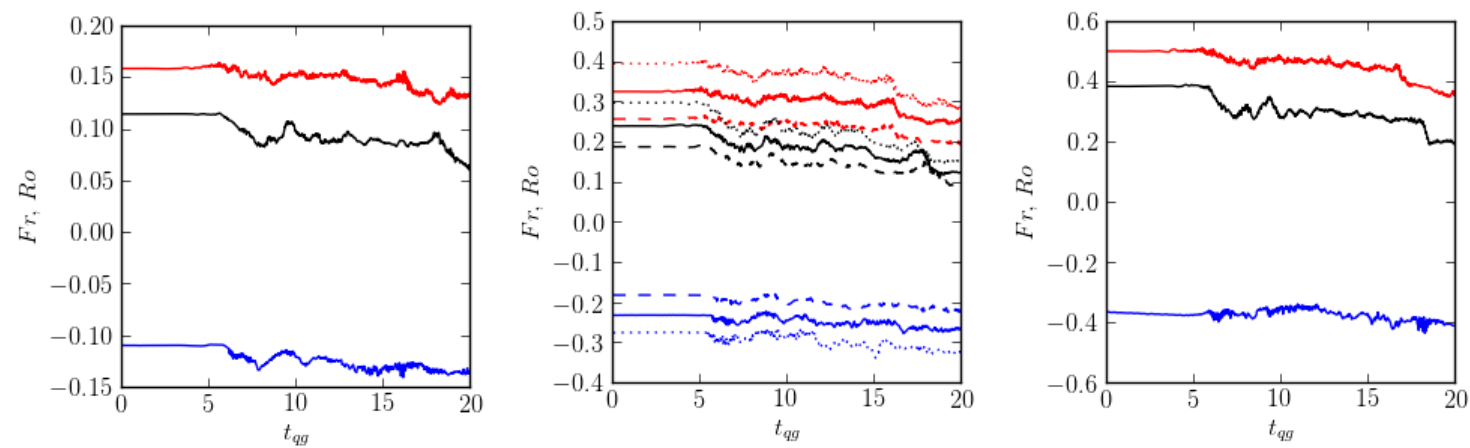

Figure 18. Non hydrostatic shielded vortex with $r_{2} / r_{1}=\sqrt[3]{2}$ and $\left|q_{1} / q_{2}\right|=1$. The vortex core is a anti-cyclone, while the outer shield has cyclonic PV. $F r^{\max }$ (black), $R o^{\min }$ (blue) and $R o^{\max }$ (red). Left: $R o_{q}=-0.125$. Middle panel: $R o_{q}=-0.2$ (dashed), -0.25 (solid), -0.3 (dotted). Right $R o_{q}=-0.375$. (Colour online).
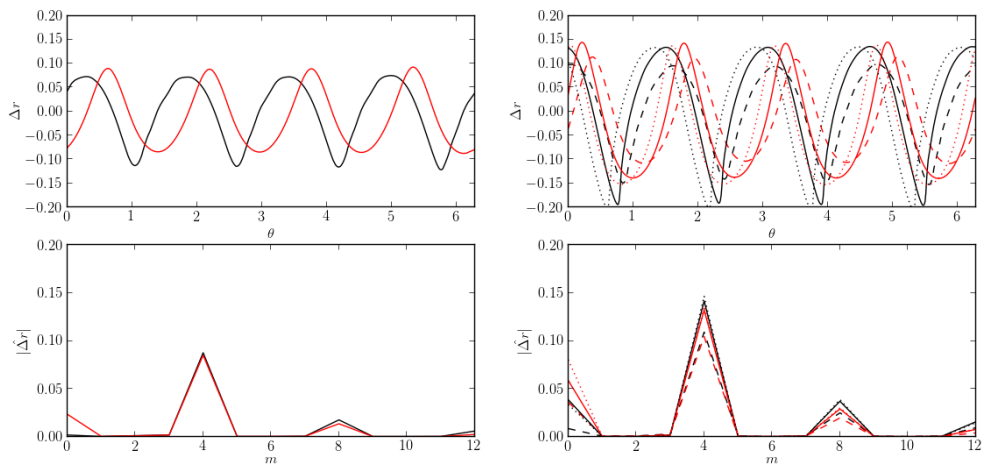

Figure 19. Non hydrostatic shielded vortex with $r_{2} / r_{1}=\sqrt[3]{2}$ and $\left|q_{1} / q_{2}\right|=1$. Deformation of the horizontal mid-section contours at $t_{q g}=5$. Inner core (red), outer shield (black). Top panels: deformation (departure of the local radius from the mean radius) in physical space $\Delta r$ vs. $\theta$. Bottom panels: Amplitude of the Fourier modes $|\hat{\Delta r}|$ vs. the azimuthal wave number $m$ (only small $m$ shown). Left $R o_{q}=0.125$, right $R o_{q}=0.2$ (dashed), 0.25 (solid), 0.3 (dotted). (Colour online).

numbers $R o^{\min }\left(\right.$ resp. $R o^{\max }$ )

$$
\begin{gathered}
\operatorname{Fr}^{\max }(t) \equiv \max _{\boldsymbol{x} \in D} \frac{\left|\boldsymbol{\omega}_{h}(\boldsymbol{x}, t)\right|}{N}, \\
R o^{\min }(t) \equiv \min _{\boldsymbol{x} \in D} \frac{\zeta(\boldsymbol{x}, t)}{f}, \quad \operatorname{Ro}^{\max }(t) \equiv \max _{\boldsymbol{x} \in D} \frac{\zeta(\boldsymbol{x}, t)}{f} .
\end{gathered}
$$

where $\boldsymbol{\omega}_{h}$ is the horizontal part of $\boldsymbol{\omega}$

The time evolution of these quantities is presented in figure 17 for $R o_{q}>0$, and in figure 18 for $R o_{q}<0$. To compare cases with different values of $\left|R o_{q}\right|$ we use an equivalent QG-time $t_{q g}=t q_{1} / N=t R o_{q}(f / N)$. Both cases indicate that the peak vertical vorticity is found in the outer shield as $\left|R o^{\min }\right|>R o^{\max }$ when $R o_{q}>0$ (resp. $R o^{\max }>\left|R o^{\min }\right|$ when $\left.R o_{q}<0\right)$. The results also show that (in general) the peak horizontal vorticity tend to decrease with time (although the time evolution is not monotonous), while the peak vertical vorticity in the core (associated $R o^{\max }$ for $R o_{q}>0,\left|R o^{\min }\right|$ for $R o_{q}<0$ ) tend to overall increase in magnitude.

We next turn our attention to the deformation of the horizontal mid-section contours at early times. Overall, all cases exhibit a main deformation for the mode $m=4$, similarly to what was observed in the (periodic) QG limit. At early stage $\left(t_{q g}=5\right)$, the case $\left|R o_{q}\right| \simeq 0.25$ exhibits the largest contour deformations. There is no significant difference between the magnitude of the deformation of the inner and the outer contours. However, for fixed $\left|R o_{q}\right|$, the deformation for the anti-cyclonic PV shield $\left(R o_{q}>0\right)$ is slightly larger than in the cyclonic shield case 

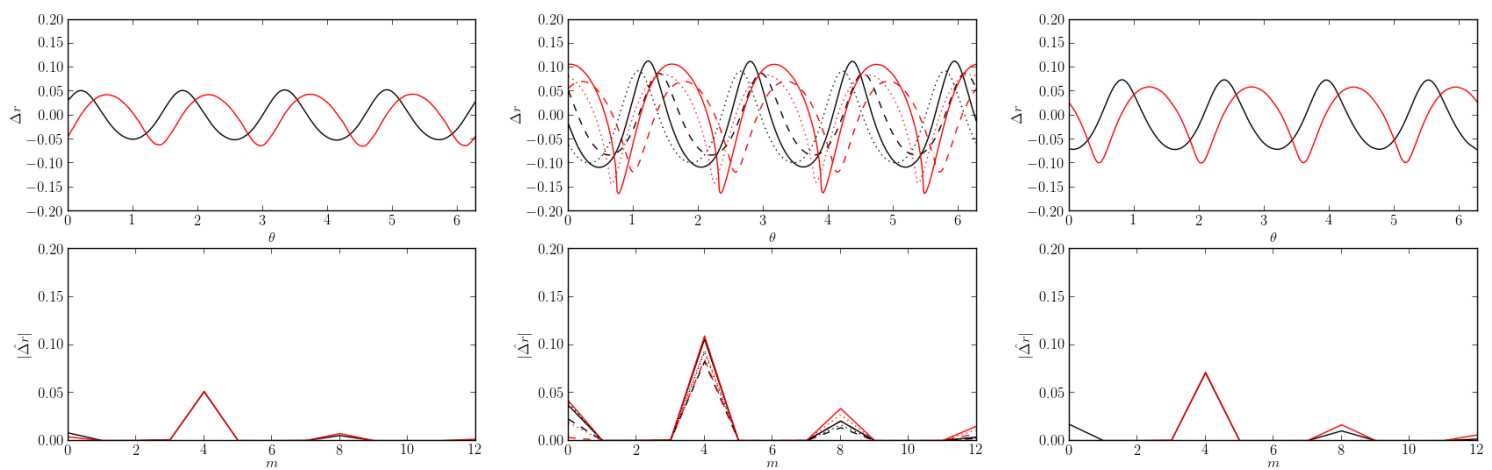

Figure 20. Non hydrostatic shielded vortex with $r_{2} / r_{1}=\sqrt[3]{2}$ and $\left|q_{1} / q_{2}\right|=1$. Deformation of the horizontal mid-section contours at $t_{q g}=5$. Inner core (red), outer shield (black). Top panels: deformation (departure of the local radius from the mean radius) in physical space $\Delta r$ vs. $\theta$. Bottom panels: Amplitude of the Fourier modes $|\hat{\Delta r}|$ vs. the azimuthal wave number $m$ (only small $m$ shown). Left $R o_{q}=-0.125$, middle $R o_{q}=-0.2$ (dashed), -0.25 (solid), -0.3 (dotted), and right $R o_{q}=-0.375$. (Colour online).

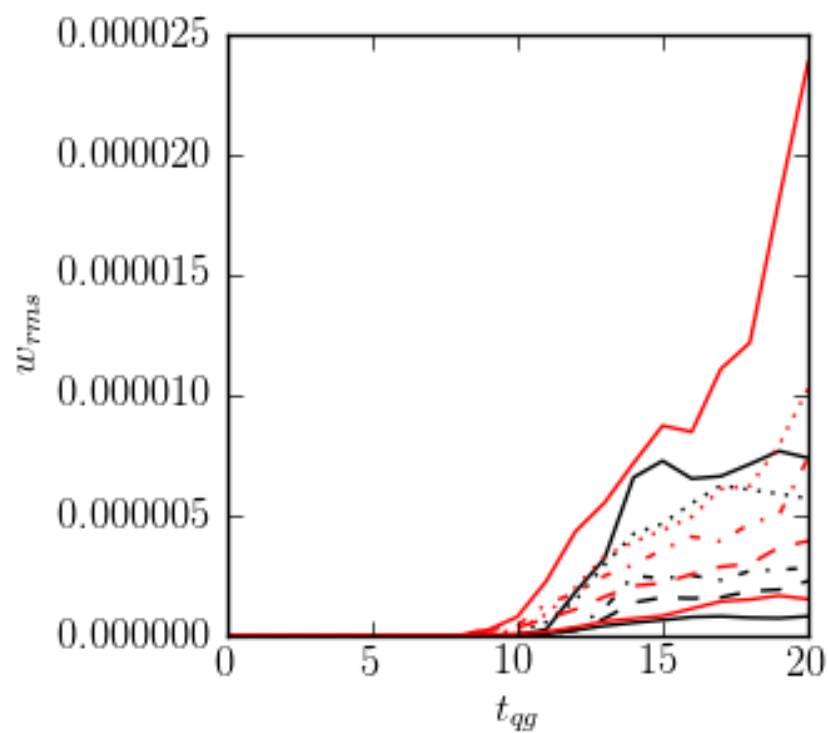

Figure 21. Vertical velocity rms $w_{r m s}$ vs. time $t_{q g}$ for $R o_{q}>0$ (red) and $R o_{q}<0$ (black). $\left|R o_{q}\right|=0.125$ (lower solid lines), 0.2 (dashed lines), 0.25 (dot-dashed lines), 0.3 (dotted lines), and 0.375 (upper solid lines). (Colour online).

$\left(R o_{q}<0\right)$.

The root mean square of the vertical velocity $w_{r m s}(t)$ in the horizontal mid-section of the computational domain is plotted vs $t_{q g}$ in figure 21 . The vertical velocities increase with $\left|R o_{q}\right|$ which is consistent with the Rossby and Froude scaling of the non-hydrostatic equations. Scaling analysis shows that the scale of vertical velocity $W$ relates to the horizontal velocity scale $U$ through $W \sim R o^{-1} \mathrm{Fr}^{2} H U / L$, where $L$ (resp. $H$ ) is a characteristic horizontal (resp. vertical) length scales. In our case, we see that $R o \sim F r$, hence $R^{-1} F r^{2} \sim R o$. It is also shown that $w_{r m s}$ is larger for $R o_{q}>0$. The vertical velocity can be seen as a indicator of inertia-gravity wave activity (although of part of $w$ is due to balanced motion). It is also seen that cases with $R o_{q}>0$ produces on average higher vertical velocities, although the velocity remains very small in amplitude.

Figures 22-27 illustrate the time evolution of the shielded vortex for $R o_{q}=$ $-0.125,0.125,-0.25,0.25,-0.375,0.375$. The figures show a top view of the vortex, a horizontal cross-section (passing thought the centre of the vortex) of the vertical vorticity $\zeta$ and 
vertical velocity $w$. The frames confirm that, at early stages, the instability grows faster for $R o_{q}>0$ as the deformation is more pronounced at $t_{q g}=5$. This is in particular visible on $\zeta$, see middle panels of figures 22-27. The right panels of figures 22-27 show that at early stages, the vertical velocity $w$ exhibit a clear wave pattern, with waves emitted by the destabilising vortex. Spontaneous emission of inertia-gravity waves by balanced (PV-controlled) motion is a well-known phenomena (see in particular Viúdez and Dristchel 2006, Viúdez 2006, Viúdez 2007, Pallàs-Sanz and Viùdez 2008) and can be due of the unsteady advection of PV.

At later stages of the flow evolution (in particular, for $R o_{q}=0.25$ ) $w$ has a spatial pattern mostly consisting of large scale features. These are likely to be associated with a balanced motion. The late evolution is highly nonlinear and therefore different in all cases run, despite the similarity of the initial conditions. This is expected in a turbulent-like evolution. However, a general pattern emerges. Mode $m=4$ dominates during the early stages, then the later evolution of the flow consists in the formation of two structures where the vorticity field has re-organised itself into dipolar structures. This is overall very similar to the QG regime discussed in the previous sections. Overall the finite Rossby and Froude numbers, at least in the range of values examined, have not affected the underlying QG dynamics significantly.

\section{2. $\quad$ Forced dynamics}

We next introduce an initial, small yet finite amplitude perturbation to the vortex by deforming the contours according to

$$
R_{i}^{\prime}(z)=R_{i}\left[1+\epsilon \cos \left(k_{f} \theta+\theta_{i}^{0}\right)\right],
$$

where $R_{i}^{2}=r_{i}^{2}-z^{2}$, is the local horizontal radius of the spherical PV jump $i=1,2, \theta$ is the local azimuthal angle, $\theta_{i}^{0}$ is a phase, and $k_{f}$ is the wavenumber of the monochromatic perturbation. The phase $\theta_{i}^{0}$ is given by the linear stability analysis of the QG case. In practice, only $\theta_{2}^{0}-\theta_{1}^{0}$ matters and is equal to $\pm \pi / 2$, the sign depending on the sign to $R o_{q}$. We set $\epsilon=0.005$. i.e. $5 \%$ of the unperturbed radius. This amplitude is large enough to guarantee that the prescribed mode dominates the dynamics. We investigate the nonlinear evolution for $k_{f}=2,3$, and 4 , for for values of the Rossby number $R o_{q}= \pm 0.125, \pm 0.25$ and \pm 0.375 . For each choice of $R o_{q}$ we therefore run 3 simulations, one for each value of $k_{f}$.

Figure 28 shows the amplitude of the Fourier modes for the deformation of the central inner contour at $t_{q g}=2$. Results, not shown, for the outer contour are similar. We see that, at early stage, the mode $k_{f}=3$ is the most amplified, followed by $k_{f}=4$, then $k_{f}=2$. This is consistent with the linear stability analysis in the QG regime. We also see that, overall, at the same rescaled time $t_{q g}$, the amplitude of the mode is only weakly dependent on $\left|R o_{q}\right|$ for given $k_{f}$. It seems however, that $k_{f}=2$, and 3 is marginally favoured for $R o_{q}<0$ while $k_{f}=4$ is favoured by $R o_{q}>0$. It should be noted however that the differences are very small. Nevertheless, if there is a weak dependence of the stability properties of the non-hydrostatic shielded vortex, it is mode-dependent.

The vertical velocity $r m s, w_{r m s}$, is plotted vs. time in figure 29 . Recall that the results are plotted against a rescaled time scale $t_{q g}$ which compensates for the value of $R o_{q}$. Hence, recall that the structure destabilises faster (in buoyancy period) for higher values of $\left|R o_{q}\right|$. It is seen again that the vertical velocities remain very small in the early stages of the destabilisation of the vortex, and only increase (while remaining small) at the late stage of the evolution flow, when the flow is governed by the complex interaction of smaller scale vortices and PV debris. Again, larger values of the Rossby number generate more vertical motion, as expected. The most noticeable differences between the cases $R o_{q}<0$ and $R o_{q}>0$ do not appear during the early stage (nearly linear regime). Differences are more pronounced at later stage of the nonlinear evolution.

Figures 30-31 provide a horizontal cross-section of the vertical component of the relative 

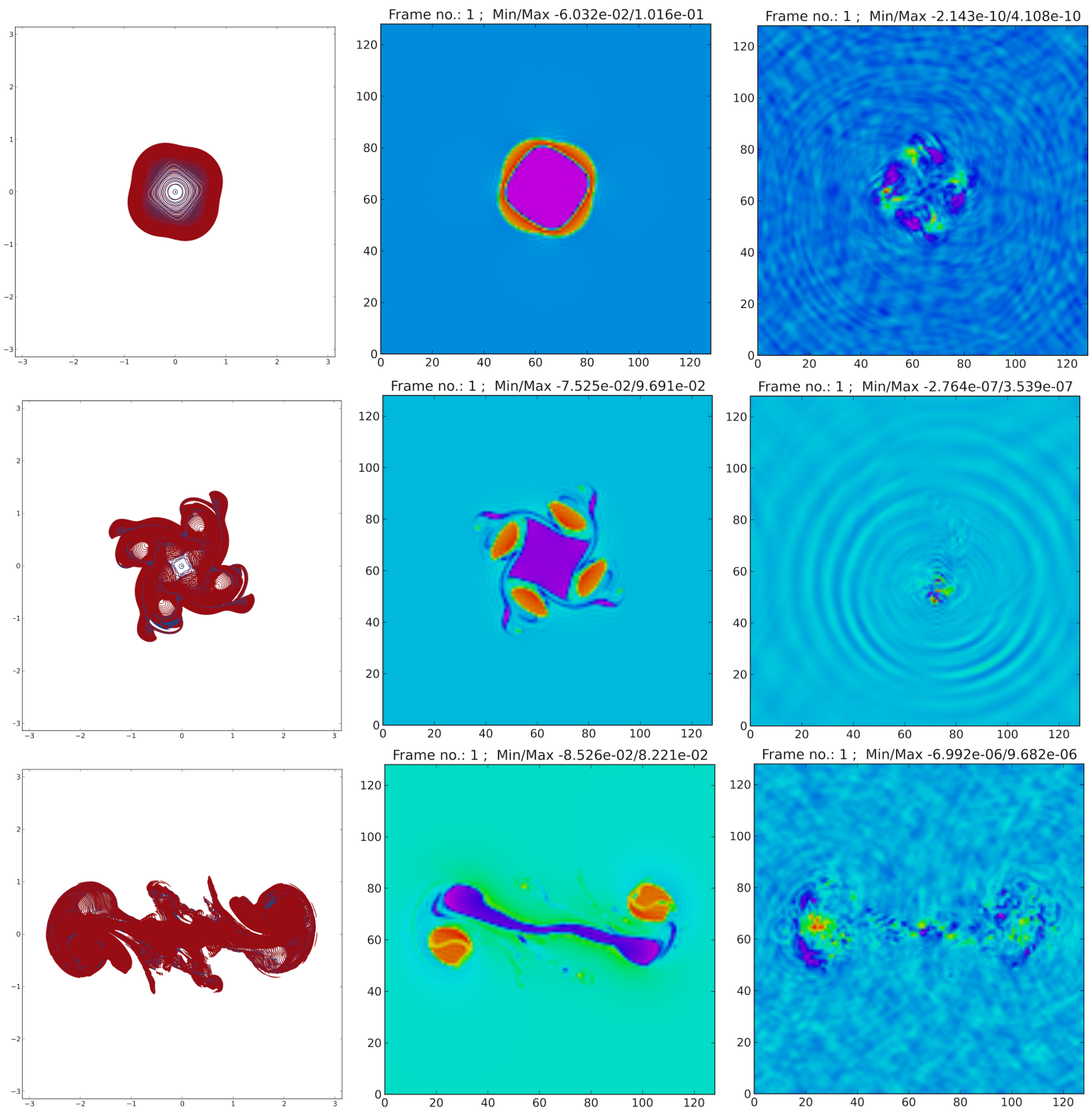

Figure 22. Non-hydrostatic spherical shielded vortex with $\left|q_{2} / q_{1}\right|=1$ and $r_{2} / r_{1}=\sqrt[3]{2}, R o_{q}=-0.125$ at, from top to bottom $t=5,10$, and 20. Left panel: Top view on the contours defining the vortex. Middle panel: vertical vorticity $\zeta$ in the horizontal mid-section of the vortex. Right panel: Vertical velocity $w$ in the horizontal mid-section of the vortex. (Colour online).

vorticity $\zeta$ in the mid-section in the structure for the two cases $R o_{q}= \pm 0.375$. We can see that the details of the time evolution differ in the two cases illustrated. This is particularly true for the cases forced with the monochromatic mode $k_{f}=2$, and $k_{f}=3$. For $k_{f}=2$ and $R o_{q}<0$, the positive $\mathrm{PV}$ from the shield tends to reorganise itself rapidly into two vortices at the edges of the now deformed inner core. It does this faster than the negative vorticity of the shield does for $R o_{q}>0$, forcing the inner core differently. For $R o_{q}<0$, the inner (negative) PV remains near the centre as a (deformed) monopole. Then, at later stage the two positive poles stemming for the shield become satellites of the central (negative PV) pole. On the other hand, for $R o_{q}>0$, and $k_{f}=2$, some of the peripheral PV of the central (positive) core reorganises as two secondary poles located nearby the (negative) PV poles stemming from the shield. These structures form dipoles which move away from the centre, elongating the central part of the inner core. For $k_{f}=3$, the shield first reorganises as three satellite poles located aound the core. For $R o_{q}>0$, more of the central, inner core PV seems to pulled away by the satellite poles generated by the destabilisation of the shield than in the counter situation with 

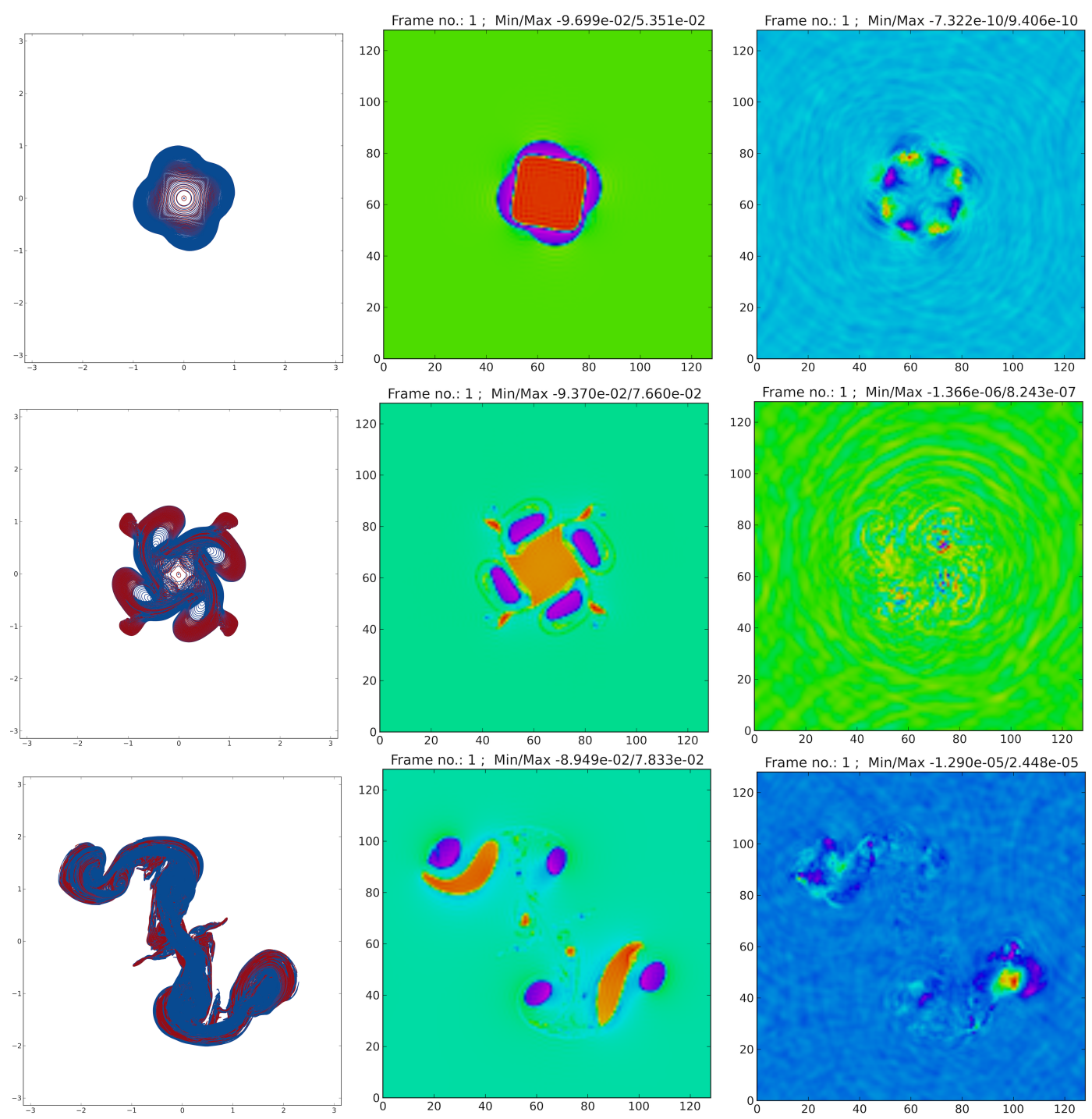

Figure 23. Non-hydrostatic spherical shielded vortex with $\left|q_{2} / q_{1}\right|=1$ and $r_{2} / r_{1}=\sqrt[3]{2}, R o_{q}=0.125$ at, from top to bottom $t=5,10$, and 20. Left panel: Top view on the contours defining the vortex. Middle panel: vertical vorticity $\zeta$ in the horizontal mid-section of the vortex. Right panel: Vertical velocity $w$ in the horizontal mid-section of the vortex. (Colour online).

$R o_{q}<0$.

For reference, the nonlinear evolution of the forced shielded vortices within the quasigeostrophic regime is proposed in the appendix B.

\section{Conclusion}

This paper has addressed the linear stability and the nonlinear evolution of piecewise uniform potential vorticity, shielded, spherical vortices. This study has focused on the situation where the overall volume-integrated PV is zero. This study has shown that such quasi-geostrophic vortices are unstable when the PV ratio $\left|q_{2} / q_{1}\right|>0.2136$ (equivalent to the radius ratio $\left.r_{2} / r_{1}<1.7844\right)$. Shielded vortices with larger radius ratio are linearly stable. The instabilities lead to the destruction of the structure, and the resulting secondary vortices do not resemble shielded vortices. 

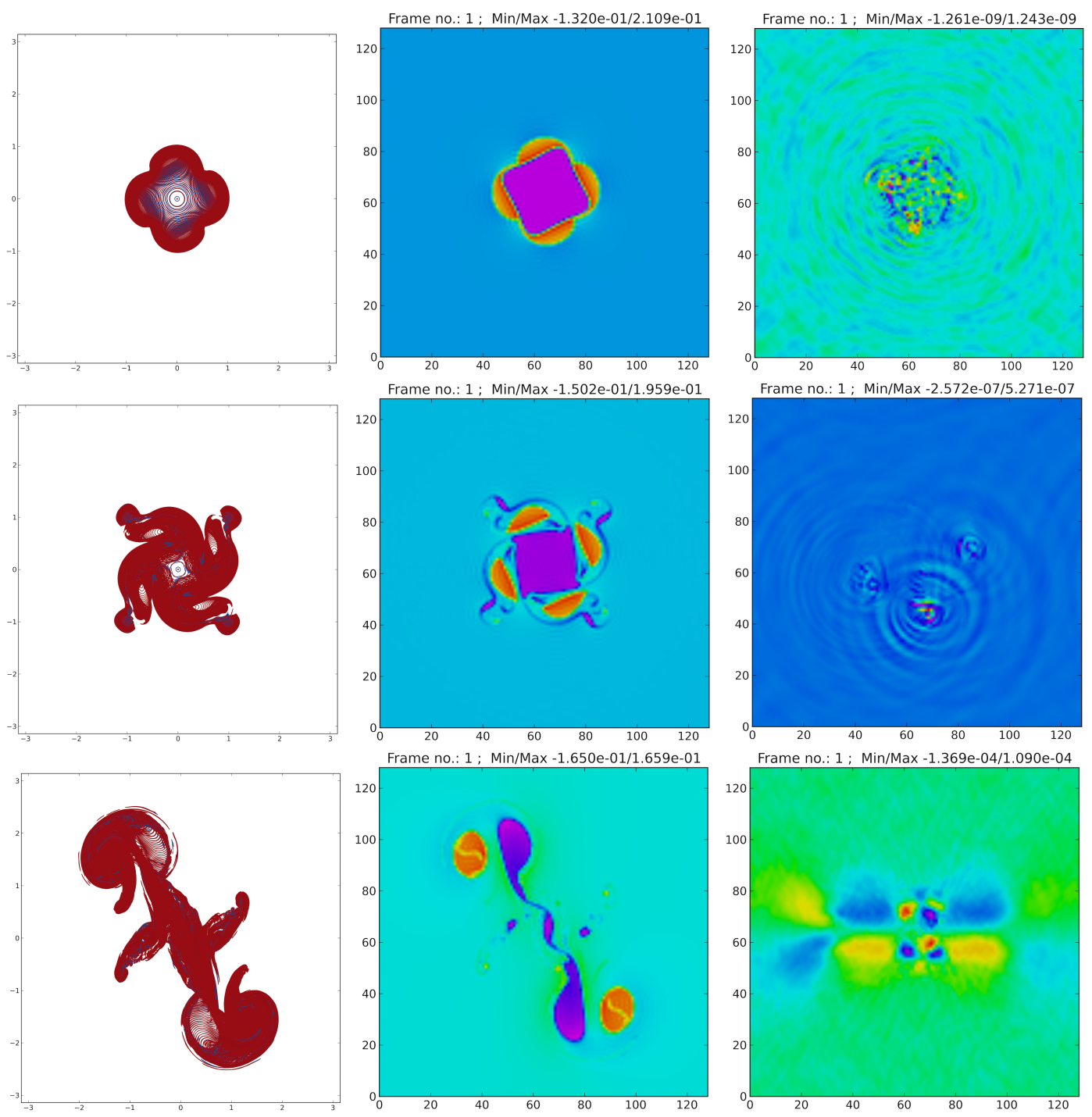

Figure 24. Non-hydrostatic spherical shielded vortex with $\left|q_{2} / q_{1}\right|=1$ and $r_{2} / r_{1}=\sqrt[3]{2}, R o_{q}=-0.25$ at, from top to bottom $t=5,10$, and 20. Left panel: Top view on the contours defining the vortex. Middle panel: vertical vorticity $\zeta$ in the horizontal mid-section of the vortex. Right panel: Vertical velocity $w$ in the horizontal mid-section of the vortex. (Colour online).

It has been confirmed that the instabilities also affect spheroidal vortices as well as laterally shielded cylindrical vortices (as expected). For spheroidal vortices, the aspect ratio has a strong impact on the nature of the deformation. This results from differences in the amplification rate of the various unstable wave numbers.

We have investigated the evolution non-hydrostatic spherical shielded vortices in the case the magnitude of the PV anomaly in the core is the same as in the shield.

Overall, the non-hydrostatic stability properties of the vortex appear similar to the QG one, at least at the early linear stages. The nonlinear regime is, on the other hand, affected by the polarity of the shielded vortex. The formation of the first secondary structures stemming from the destabilisation of the shield influences the way the whole structure evolves at later stages. This stage depends also on the mode amplified, which sets the number of secondary satellite cores generated around the inner core, as well as their topology. This, in turn, influences in a non trivial way, the further evolution of the inner core. In other words, the nonlinear interaction shows the interplay between the basic balanced dynamics of the underlying 

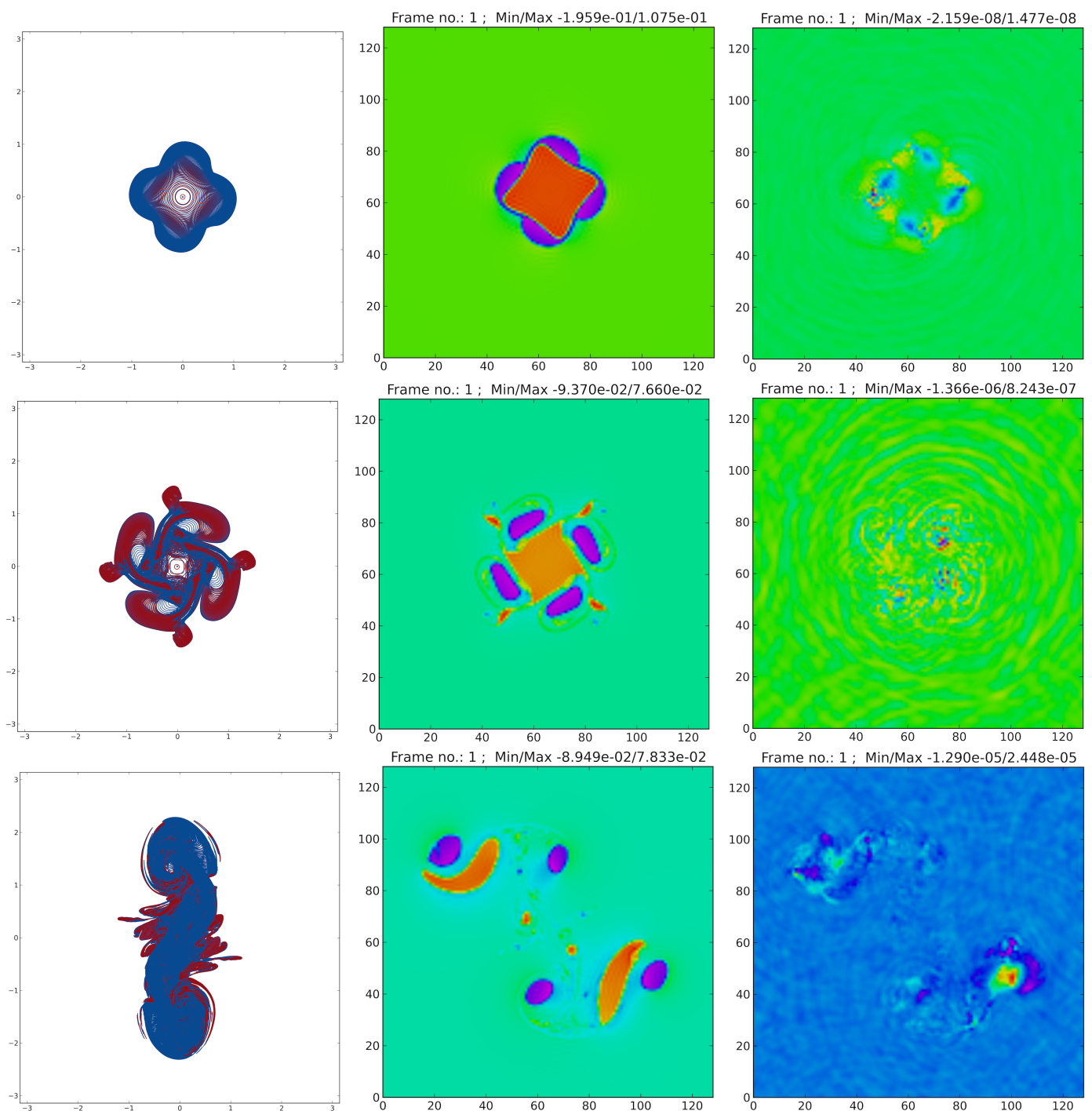

Figure 25. Non-hydrostatic spherical shielded vortex with $\left|q_{2} / q_{1}\right|=1$ and $r_{2} / r_{1}=\sqrt[3]{2}, R o_{q}=0.25$ at, from top to bottom $t=5,10$, and 20. Left panel: Top view on the contours defining the vortex. Middle panel: vertical vorticity $\zeta$ in the horizontal mid-section of the vortex. Right panel: Vertical velocity $w$ in the horizontal mid-section of the vortex. (Colour online).

PV-controlled destabilisation (which is close to the QG dynamics) and dissymmetrization of behaviour (cyclonic/anti-cyclonic PV) associated with the ageostrophic part of the dynamics.

Further investigation of vortices in the non-hydrostatic regime is necessary. For example, Sutyrin and Radko (2016) recently showed that Gaussian vortices (which have vertical vorticity of both sign) can be stabilised by adapting the stratification and enforcing that the PV is single-signed.

\section{References}

Benilov, E., Stability of vortices in a two-layer ocean with uniform potential vorticity in the lower layer. J. Fluid Mech. 2004, 502, 207-232.

Carton, X.J., On the merger of shielded vortices. Europhys. Lett. 1992, 18, 697-703.

Carton, X. and Legras, B., The life-cycle of tripoles in two-dimensional incompressible flows. J. Fluid Mech. 1994, 267, 53-82

Carton, X., Hydrodynamical modeling of oceanic vortices Surveys in Geophysics 2001, 22(3), 179-263. 

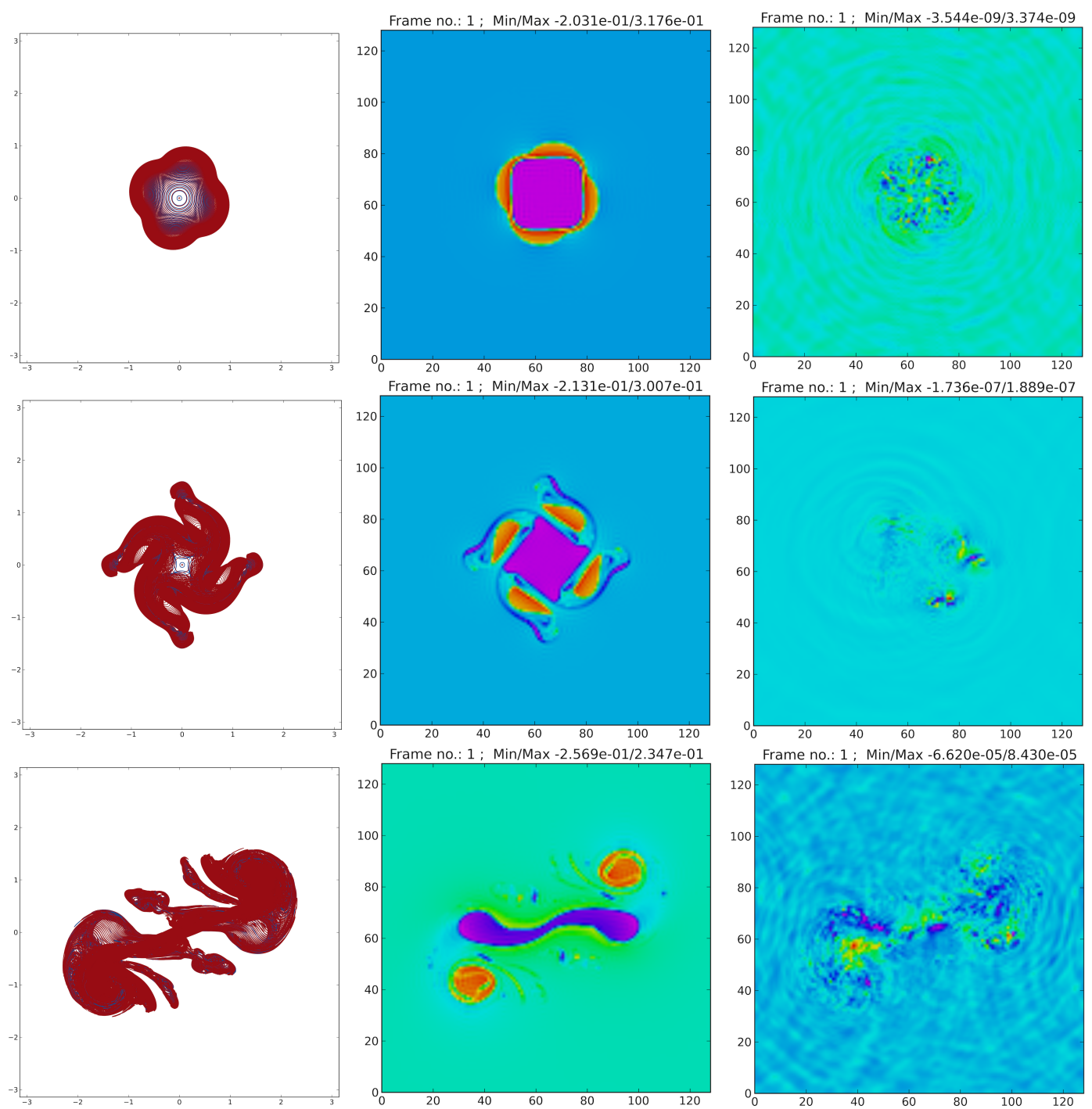

Figure 26. Non-hydrostatic spherical shielded vortex with $\left|q_{2} / q_{1}\right|=1$ and $r_{2} / r_{1}=\sqrt[3]{2}, R o_{q}=-0.375$ at, from top to bottom $t=5,10$, and 20. Left panel: Top view on the contours defining the vortex. Middle panel: vertical vorticity $\zeta$ in the horizontal mid-section of the vortex. Right panel: Vertical velocity $w$ in the horizontal mid-section of the vortex. (Colour online).

Chelton, D.B., Schlax, M.G., Samelson, R.M. and de Szoeke, R.A., Global observations of large oceanic eddies. Geophys. Res. Lett. 2007, 34, L15606.

Chelton, D.B., Schlax, M.G. and Samuelson, R.M., Global observations of nonlinear mesocale eddies. Prog. Oceanogr. 2011, 91, 167-216.

Dritschel, D.G., Nonlinear stability bounds for inviscid, two-dimensional, parallel or circular flows with monotonic vorticity, and the analogous three-dimensional quasi-geostrophic flows. J. Fluid Mech. 1988, 191, $575-582$.

Dritschel, D.G., A fast contour dynamics method for many-vortex calculations in two-dimensional flows. Phys. Fluids A 1993, 5(1), 173-186.

Dritschel, D.G. and Ambaum, M.H.P., A contour-advective semi-Lagrangian numerical algorithm for simulating fine-scale conservative dynamical fields. Q. J. Roy. Meteor. Soc. 1997, 123, 1097-1030.

Dritschel, D.G. and McKiver, W.J., Effect of Prandtl's ratio on balance in geophysical turbulence, J. Fluid Mech. 2015, 777, 569-590.

Dristchel, D.G., Scott, R.K. and Reinaud, J.N., The stability of quasi-geostrophic ellipsoidal vortices J. Fluid Mech. 2005, 536, 401-421.

Dritschel, D.G. and Viùdez, A., A balanced approach to modelling rotating stably-stratified geophysical flows J. Fluid Mech. 2003, 488, 123-150.

Ebbesmeyer, C.C., Taft, B.A., McWilliams, J.C., Shen, C.Y., Riser, S.C., Rossby, H.T., Biscaye, P.E. and Östlund, H.G., Detection, structure and origin of extreme anomalies in a Western Atlantic oceanographic 

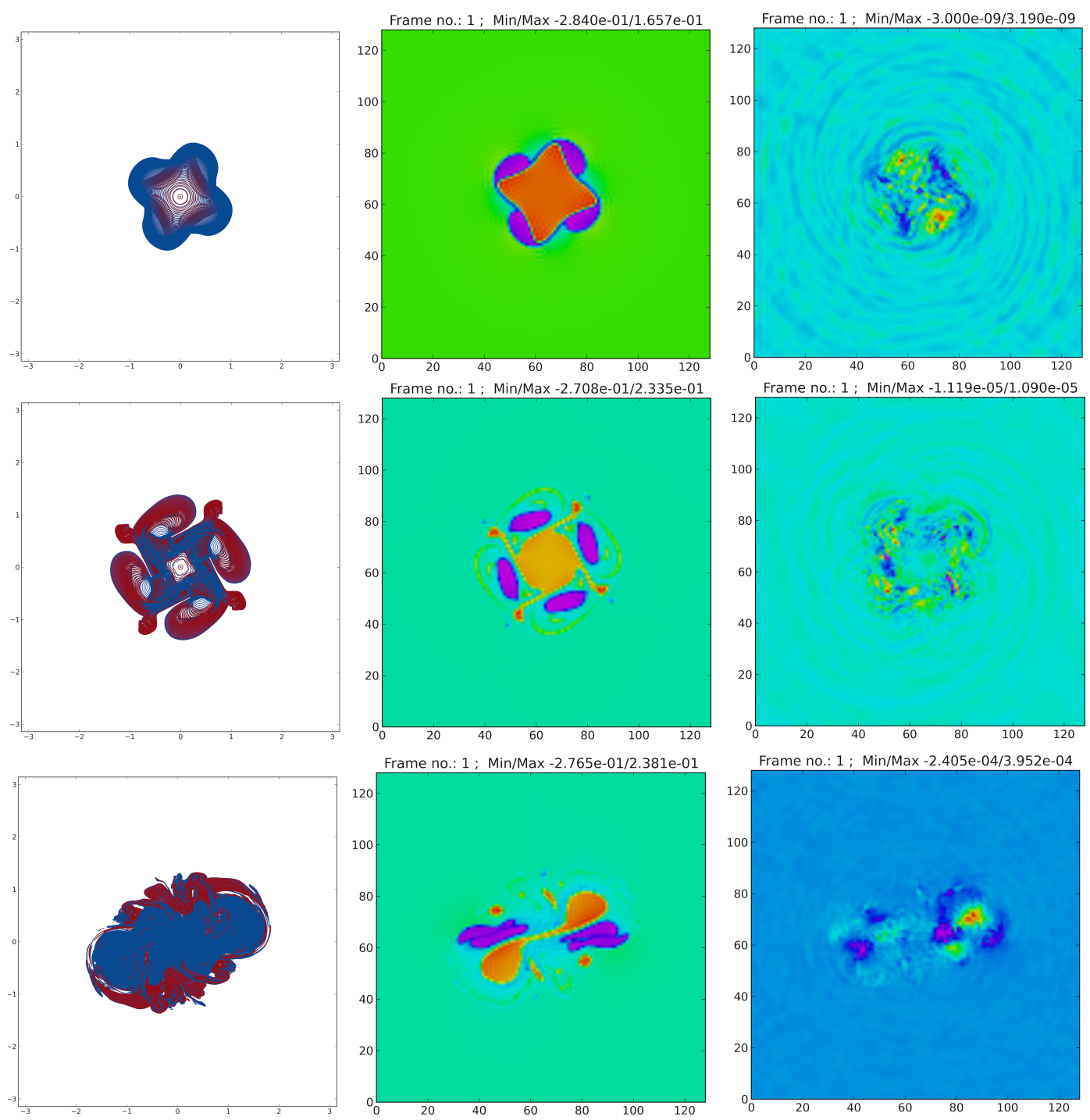

Figure 27. Non-hydrostatic spherical shielded vortex with $\left|q_{2} / q_{1}\right|=1$ and $r_{2} / r_{1}=\sqrt[3]{2}, R o_{q}=0.375$ at, from top to bottom $t=5,10$, and 20. Left panel: Top view on the contours defining the vortex. Middle panel: vertical vorticity $\zeta$ in the horizontal mid-section of the vortex. Right panel: Vertical velocity $w$ in the horizontal mid-section of the vortex. (Colour online).

section J. Phys. Ocean. 1986, 16, 591-612.

Flierl, G.R., On the instability of geostrophic vortices. J. Fluid Mech. 1988, 197, 3490-388.

Harvey, B.J., Ambaum, M.H.P. and Carton, X.J., Instability of shielded surface temperature vortices. J. Atmos. Sci. 2011, 68, 964-971.

Mariotti, A., Legras, B. and Dritschel, D.G., Vortex stripping and the erosion of coherent structures in twodimensional flows. Phys. Fluids 1994, 6, 3954-3962.

McWililams, J.C., Submesoscale, coherent vortices in the ocean Rev. Geophys. 1985, 22, 165-182.

Meacham, S.P., Quasigeostrophic, ellipsoidal vortices in stratified fluid. Dyn. Atmos. Oceans 1992, 16, $189-223$.

Miyazaki, T., Ueno, K. and Shimonishi, T., Quasigeostrophic tilted spheroidal vortices. J. Phys. Soc. Japan 1999, 68, 2592-2601.

Miyazaki, T., Fujiwara, T. and Yamamoto, M., Quasigeostrophic confocal spheroidal vortices. J. Phys. Soc. Japan 2003, 72, 2786-2803.

Morel, Y.G. and Carton, X.J., Multipolar vortices in two-dimensional incompressible flows. J. Fluid Mech. 1994, 267, 23-51.

Morel, Y. and McWilliams, J., Evolution of isolated vortices in the ocean. J. Phys. Oceanogr. 1997, 27, 727-748. Pallàs-Sanz, E. and Viúdez, A., Spontaneous generation of inertia-gravity waves during the merging of two baroclinic anticyclones. J. Phys. Oceanogr. 2008, 38, 213-234.

Reinaud, J.N., On the stability of continuously stratified quasi-geostrophic hetons Fluid Dyn. Res. 2015, 43, 

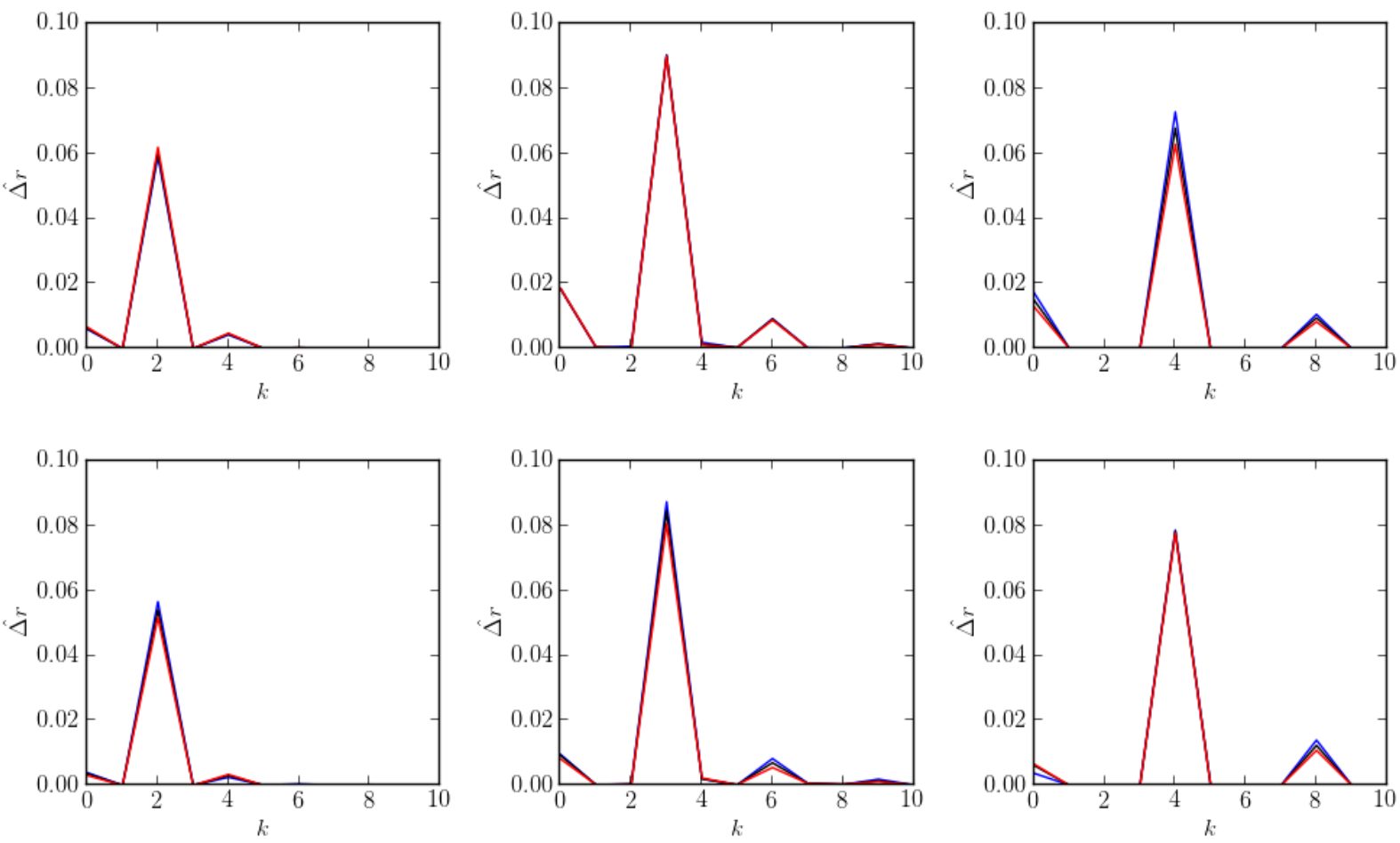

Figure 28. Non-hydrostatic spherical shielded vortex with $\left|q_{2} / q_{1}\right|=1$ and $r_{2} / r_{1}=\sqrt[3]{2}$ : Fourier modes of the deformation of the central inner contour at $t_{q g}=2$ for $k_{f}=2$ (left), $k_{f}=3$ (centre), and $k_{f}=4$ (right), and $R o_{q}<0$ (top), $R o_{q}>0$ (bottom). $\left|R o_{q}\right|=0.125$ (blue), 0.25 (black), and 0.375 (red). (Colour online).
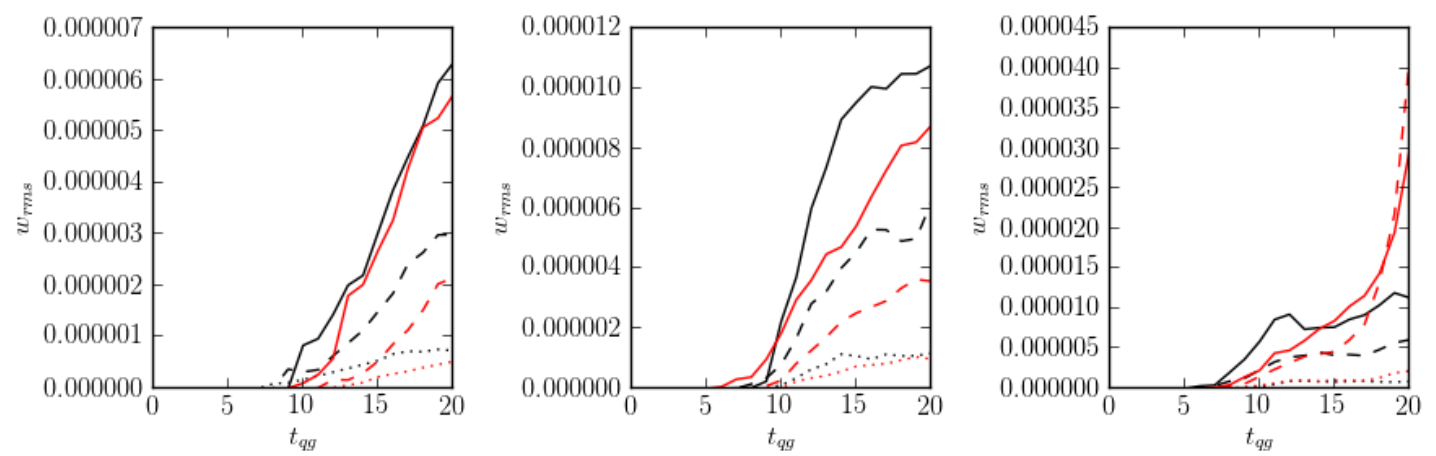

Figure 29. Non-hydrostatic spherical shielded vortex with $\left|q_{2} / q_{1}\right|=1$ and $r_{2} / r_{1}=\sqrt[3]{2}$ : Root mean square of the vertical velocity $w_{r m s}$ over the three-periodic domain vs. $t_{q g}$ for $k_{f}=2$ (left panel), 3 (central panel), and 4 (right panel). Red curves correspond to $R o_{q}<0$ while black curves are for $R o_{q}>0$. and $\left|R o_{q}\right|=0.125$ (dotted line), 0.25 (dashed line), 0.375 (solid line). (Colour online).

035510.

Reinaud, J.N. and Carton, X., The stability and the nonlinear evolution of quasi-geostrophic hetons J. Fluid Mech. 2009, 636, 109-135.

Reinaud, J.N. and Carton, X., Existence, stability and formation of baroclinic tripoles in quasi-geostrophic flows J. Fluid Mech. 2015, 785, 1-30.

Reinaud, J.N. and Dritschel, D.G., The merger of vertically offset quasi-geostrophic vortices J. Fluid Mech. 2002, 469, 287-315.

Reinaud, J.N. and Dritschel, D.G., Destructive interactions between two counter-rotating quasi-geostrophic vortices J. Fluid Mech. 2009, 639, 195-211.

Reinaud, J.N., Dritschel, D.G. and Koudella, C.R., The shape of vortices in quasi-geostrophic turbulence. J. Fluid Mech. 2003, 474, 175-192.

Robinson, A.R., Eddies in Marine Science 1983, Springer Verlag. 

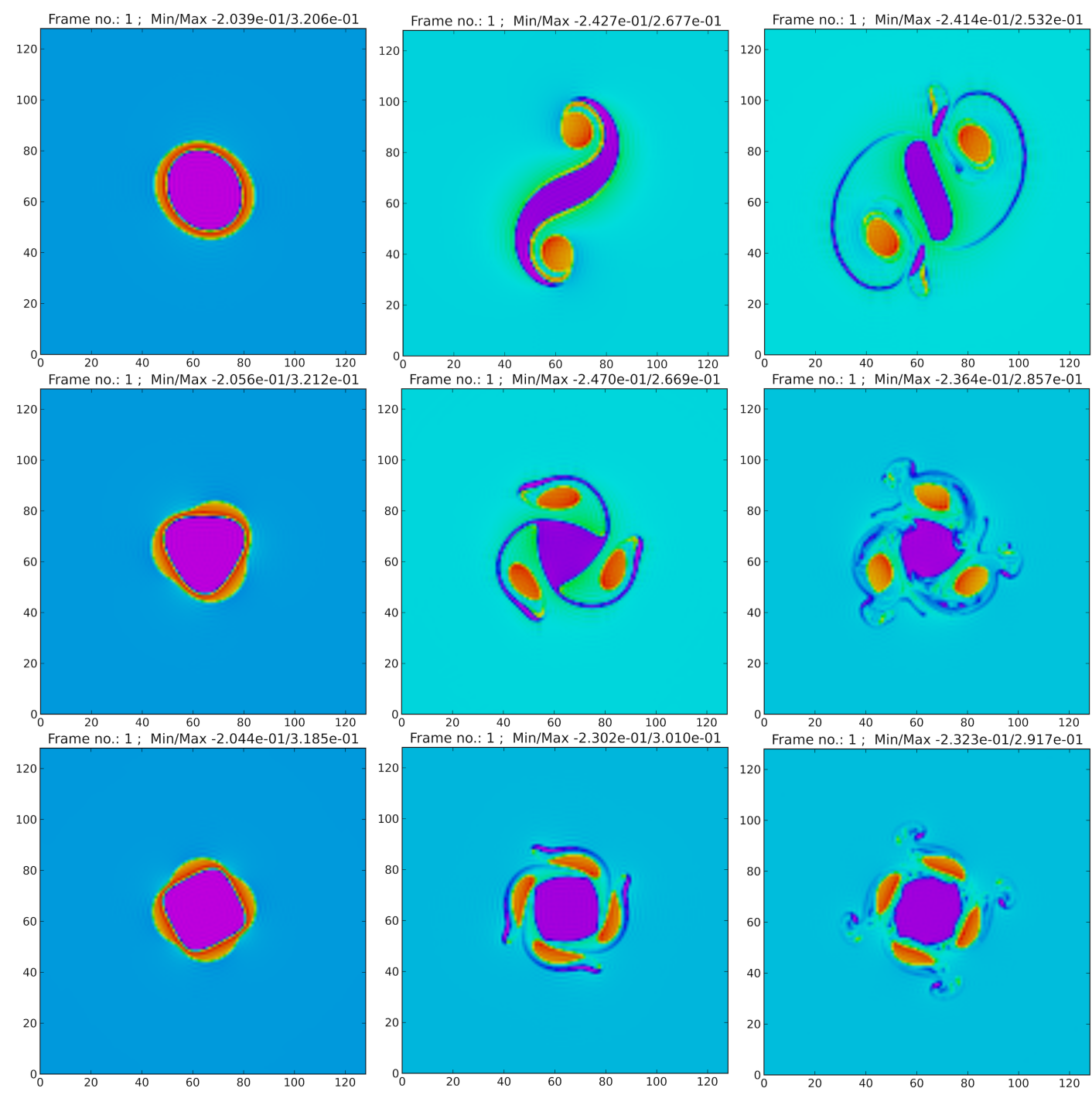

Figure 30. Non-hydrostatic spherical shielded vortex with $\left|q_{2} / q_{1}\right|=1$ and $r_{2} / r_{1}=\sqrt[3]{2}$ : Horizontal mid cross-section of the vertical component of relative vorticity $\zeta$ for $R o_{q}=-0.375$, and $k_{f}=2$ (top row), 3 (middle row), 4 (bottom row). Times displayed are $t_{q g}=2$ (left column), 5 (central column), and 10 (right column). (Colour online).

Sutyrin, G., Why cold core rings look stable? Geophys. Res. Lett. 2015, 42, 5395-5402.

Sutyrin, G. and Radko, T., Stabilization of isolated vortices in a rotating stratified fluid Fluids 2016, doi:10.3390/fluids1030026.

Tsang, Y.-K. and Dritschel, D.G., Ellipsoidal vortices in rotating stratified fluids: beyond the quasi- geostrophic approximation. J. Fluid Mech. 2015, 762, 196-231.

van Heijst, G.J.F. and Clercx, H.J.H., Laboratory modeling of geophysical vortices. Annu. Rev. Fluid Mech. 2009, 41, 143-164.

Viúdez, A., Spiral patterns of inertiagravity waves in geophysical flows. J. Fluid Mech. 2006, 562, 73-82.

Viúdez, A., On the mass anomaly of potential vorticity vortices (2015) Q. J. Roy. Meteor. Soc. 2015, 141(692), $2518-2527$.

Viúdez, A. and Dritschel, D.G., Spontaneous generation of inertia-gravity wave packets by balanced geophysical flows J. Fluid Mech. 2006, 553, 107-117.

Yim, E. and Billiant, P., Analogies and differences between the stability of an isolated pancake vortex and a columnar vortex ins a stratified fluid. J. Fluid Mech. 2016, 796, 732-766.

Zhang, Z., Wang, W. and Qiu, B., Oceanic mass transport by mesocale eddies. Science 2014, 345, 322-324. 

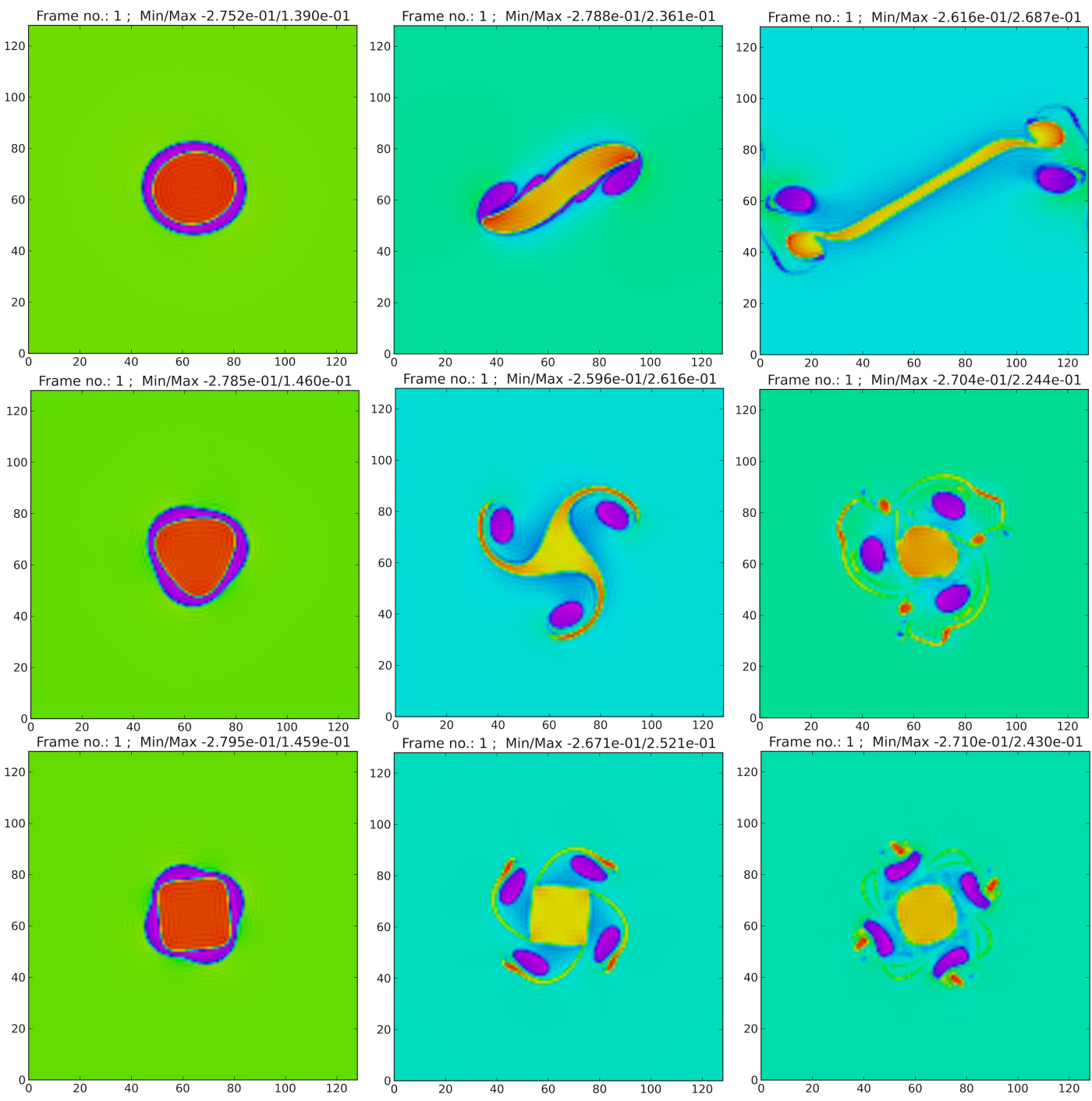

Figure 31. Non-hydrostatic spherical shielded vortex with $\left|q_{2} / q_{1}\right|=1$ and $r_{2} / r_{1}=\sqrt[3]{2}$ : Horizontal mid cross-section of the vertical component of relative vorticity $\zeta$ for $R o_{q}=0.375$, and $k_{f}=2$ (top row), 3 (middle row), 4 (bottom row). Times displayed are $t_{q g}=2$ (left column), 5 (central column), and 10 (right column). (Colour online).

\section{Appendix A: Quasi-geostrophic shielded spheroid with $h / r=2$}

We briefly illustrate the stability and the nonlinear evolution of the spheroid with $h / r=2$, for $r_{2} / r_{1}=2$. The most unstable mode is a mode $m=1$ and is non-destructive. However, the second mode is. The spatial structure for the mode is given in figure A1, and are referred to M2I for isolated vortices (see Dritschel, Scott and Reinaud 2005, figure 3, top left).

We perform a Contour surgery simulation of the vortex. As mentioned, the first mode is a mode $m=1$ and is non destructive. The second is and breaks the vortex in the vertical, see, figure A1(right).

\section{Appendix B: Forced quasi-geostrophic shielded vortices}

For illustration purposes, we provide in this appendix time snapshots of the evolution of the forced shielded vortex in the quasi-geostrophic regime. The simulations are run with the three-periodic quasi-geostrophic CASL algorithm, and the initial conditions are the same PV 

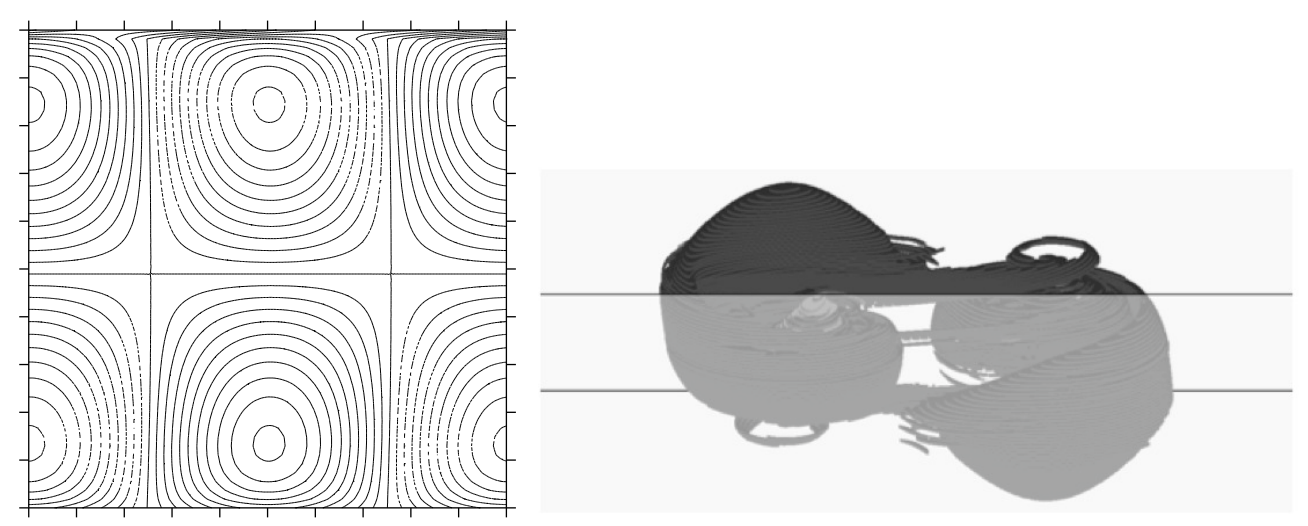

Figure A1. Left: Reconstructed deformation mode on the boundary of the inner vortex for secondary unstable modes (ordered from left to right from most to least) for the piecewise uniform shielded vortex, with $q_{1} / q_{2}=-7, r_{2} / r_{1}=2$, and $h / r=2$ The vertical axis of each frame corresponds to the vertical coordinate along the inner vortex surface, and the horizontal axis is the azimuthal angle around the vortex surface. The lines correspond to contours of iso-value of deformation amplitude. Right: snapshot of the vortex at $t=100$. The vortices are viewed orthographicaly at an angle of $75^{\circ}$ from the vertical.

contours as the ones used for the non-hydrostatic simulations.

Figure B1 shows the time evolution of the shielded vortex for the three simulations with $k_{f}=$ 2,3 , and 4 respectively. Qualitatively the evolution of the flow is similar to the non-hydrostatic case. Satellite multipoles are formed, and their number depends on the wavenumber of the perturbation. A central structure remains and is strongly affected by the shear and straining field associated with the satellite vortices. 

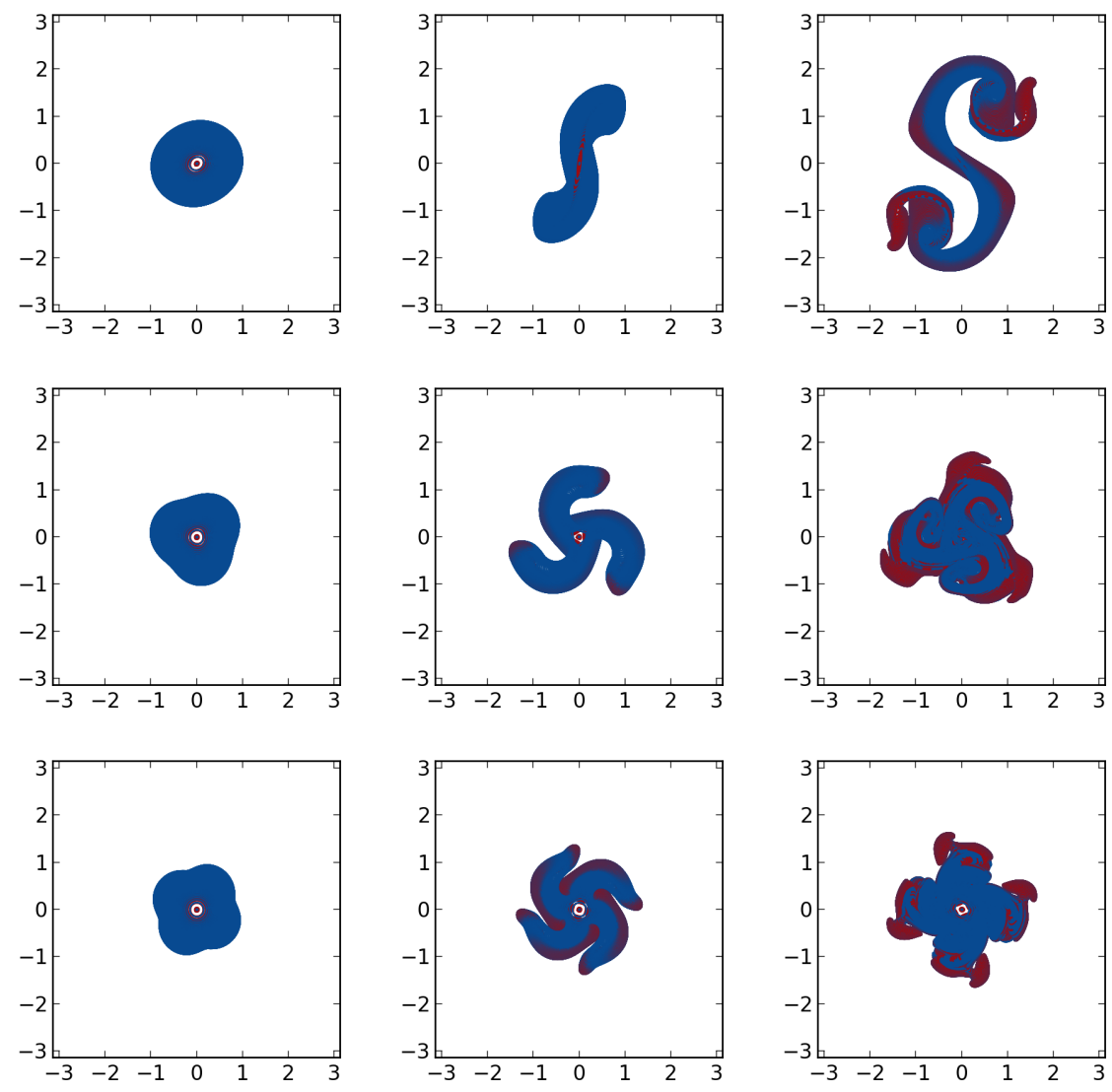

Figure B1. Top view on the PV contours defining the quasi-geostrophic shielded vortex with $\left|q_{2} / q_{1}\right|=1$ and $r_{2} / r_{1}=\sqrt[3]{2}$, and for $k_{f}=2$ (top row), 3 (middle row), and 4 (bottom row). The time displayed are $t_{q g}=2,5,10$ as in figures $30-31$. (Colour online). 